Article

\title{
Synthesis and Biological Activity of Novel O-Alkyl Derivatives of Naringenin and Their Oximes
}

\author{
Joanna Kozłowska $^{1, *}$ (D), Barłłomiej Potaniec ${ }^{1}$ (D), Barbara Żarowska ${ }^{2}$ and Mirosław Anioł ${ }^{1}$ \\ 1 Department of Chemistry, Wrocław University of Environmental and Life Sciences, Norwida 25, \\ 50-375 Wrocław, Poland; b.potaniec@gmail.com (B.P.); miroslaw.aniol@upwr.edu.pl (M.A.) \\ 2 Department of Biotechnology and Food Microbiology, Wrocław University of Environmental and Life \\ Sciences, Chełmońskiego 37/41, 51-630 Wrocław, Poland; barbara.zarowska@upwr.edu.pl \\ * Correspondence: joannakozlowska3@gmail.com; Tel.: +48-71-320-5010
}

Received: 13 August 2017; Accepted: 2 September 2017; Published: 6 September 2017

\begin{abstract}
O-Alkyl derivatives of naringenin (1a-10a) were prepared from naringenin using the corresponding alkyl iodides and anhydrous potassium carbonate. The resulting products were used to obtain oximes $(\mathbf{1 b}-\mathbf{1 0 b})$. All compounds were tested for antimicrobial activity against Escherichia coli ATCC10536, Staphylococcus aureus DSM799, Candida albicans DSM1386, Alternaria alternata CBS1526, Fusarium linii KB-F1, and Aspergillus niger DSM1957. The resulting biological activity was expressed as the increase in optical density $(\triangle \mathrm{OD})$. The highest inhibitory effect against $E$. coli ATCC10536 was observed for 7,4'-di-O-pentylnaringenin (8a), 7-O-dodecylnaringenin (9a), naringenin oxime (NG-OX), 7,4'-di-O-pentylnaringenin oxime (8b), and 7-O-dodecylnaringenin oxime $(\mathbf{9 b})(\triangle \mathrm{OD}=0)$. 7-O-dodecylnaringenin oxime $(\mathbf{9 b})$ also inhibited the growth of S. aureus DSM799 $(\Delta \mathrm{OD}=0.35)$ and C. albicans DSM1386 $(\triangle \mathrm{OD}=0.22)$. The growth of $A$. alternata CBS1526 was inhibited as a result of the action of 7,4'-di-O-methylnaringenin (2a), 7-O-ethylnaringenin (4a), 7,4'-di-O-ethylnaringenin (5a), 5,7,4'-tri-O-ethylnaringenin (6a), 7,4'-di-O-pentylnaringenin (8a), and 7-O-dodecylnaringenin (9a) ( $\triangle \mathrm{OD}$ in the range of $0.49-0.42)$ in comparison to that of the control culture ( $\triangle \mathrm{OD}=1.87)$. In the case of $F$. linii KB-F1, naringenin (NG), 7,4'-di-O-dodecylnaringenin (10a), 7-O-dodecylnaringenin oxime (9b), and 7, $4^{\prime}$-di-O-dodecylnaringenin oxime $(\mathbf{1 0 b})$ showed the strongest effect $(\triangle \mathrm{OD}=0)$. 7, $4^{\prime}$-Di-O-pentylnaringenin (8a) and naringenin oxime (NG-OX) hindered the growth of $A$. niger $\operatorname{DSM} 1957(\triangle \mathrm{OD}=0)$.
\end{abstract}

Keywords: naringenin; $O$-alkyl derivatives; oximes; antimicrobial activity

\section{Introduction}

Flavonoids are polyphenolic compounds, which are widespread in plants and food. This group comprises flavones, flavanones, flavonols, isoflavones, anthocyanidins and flavanols [1]. In plants, flavonoids usually occur in glycoside form [2,3]. Naringin is the 7-rhamno-glucoside of naringenin, which is one of the most popular flavonoids present in citrus fruits. The presence of such glycoside derivatives of flavonoids is responsible for the bitter taste of grapefruit juice [4].

In the present paper, the most interesting substrate was naringenin (4',5,7-trihydroxyflavanone), which possesses a wide spectrum of biological activities including antibacterial, antifungal, antioxidant, and anticancer activities [5,6]. Currently, there are known $O$-alkyl derivatives of naringenin containing methyl and ethyl groups attached to ring A and B. Naringenin and ether derivatives of naringenin were observed in plant extracts of the Boraginaceae family. In particular, 5-O-methylnaringenin, 7,4'-di-O-methylnaringenin and sakuranetin (7-O-methylnaringenin) (Figure 1) were isolated from Cordia globosa, Echiochilon fruticosum, Heliotropium indicum, Heliotropium stenophyllum and Corymbia torelliana $[7,8]$. Sakuranetin, which is present in rice plants, is a natural phytoalexin and provides 
effective protection against damage caused by microorganisms [9]. Moreover, it possesses anti-inflammatory activity and prevents vascular and parenchymal changes [10]. Derivatives with a benzyl group at the C-7 position regulate apoptosis in human colorectal carcinoma (RKO) cells as a result of the intracellular production of reactive oxygen species (ROS) [11]. Furthermore, 7-O-butylnaringenin exhibits antibacterial activity against methicillin-resistant Staphylococcus aureus (MRSA), which is one of the most important pathogens in hospitals [12].<smiles>O=C1CC(c2ccc(O)cc2)Oc2cc(OCC(OCC(O)O)C(O)OC(O)CO)cc(O)c21</smiles>

Naringin<smiles>COc1ccc(C2CC(=O)c3c(O)cc(OC)cc3O2)cc1</smiles>

7,4'-Di-O-methylnaringenin<smiles>O=C1CC(c2ccc(O)cc2)Oc2cc(O)cc(O)c21</smiles>

Naringenin<smiles>COc1cc(O)cc2c1C(=O)CC(c1ccc(O)cc1)O2</smiles>

5-O-methylnaringenin<smiles>COc1cc(O)c2c(c1)OC(c1ccc(O)cc1)CC2=O</smiles>

7-O-methylnaringenin (sakuranetin)

Figure 1. Structures of naringin, naringenin and naringenin derivatives.

Oxime derivatives possess very promising biological properties, e.g., antifungal [13,14], antioxidant [15-17], anticancer [18-20] and antiplatelet activities [21]. Modified flavonoids from Kaempferia parviflora exhibit antiproliferative activity toward epidermoid carcinoma of oral cavity (KB) and human small cell lung cancer (NCI-H187) cell lines about seven times higher than the analogue without the $=\mathrm{NOH}$ group [22]. Moreover, cytotoxicity assays performed on rat pheochromocytoma cell lines (PC-12), and additionally on human colon (HT-29) and breast (MCF-7) cancer cell lines, show that the oxime group increases the inhibitory effect for proliferation [18].

Current knowledge of $O$-alkyl derivatives reveals the diversity of their biological activities [7-12,23]. Our study was focused on the efficient synthesis of novel O-alkyl derivatives of naringenin and their oximes, which have not been mentioned in the literature. In the presented study, the focus is on their antimicrobial activity against different strains of bacteria and fungi. Our research proved that elongation of the $O$-alkyl chain at the $\mathrm{C}-7$ and $\mathrm{C}-4^{\prime}$ positions in naringenin leads to a significant increase in the biological activity of the obtained compounds. In addition, our studies allow the determination of the influence of introduction of the oxime group on the growth of some pathogenic strains of bacteria and fungi and compared it with the results for $O$-alkyl derivatives.

\section{Results and Discussion}

O-Alkyl derivatives were obtained by a one-step synthesis from naringenin using the appropriate alkyl iodide in the presence of potassium carbonate (1a-10a). First, reactions were performed in anhydrous acetone at room temperature for 24-96 h, which afforded a mixture of 7-O-alkyl- (1a, 4a, 7a, 9a) and 7,4'-di-O-alkylnaringenin $(\mathbf{2 a}, \mathbf{5 a}, \mathbf{8 a}, \mathbf{1 0 a})$. When using $N, N$-dimethylformamide (DMF) as the solvent, and after $7 \mathrm{~h}$ of reaction, 5,7,4'-tri-O-alkylnaringenin (3a, $\mathbf{6 a})$ was obtained. In the second step, which involved the reaction with hydrochloride hydroxylamine and anhydrous sodium acetate in ethanol, naringenin analogues were transformed into oximes (1b-10b) (Scheme 1). All crude products were purified by column chromatography, and their purity was analysed by high-performance liquid chromatography (HPLC). 
<smiles>CC(C)C1CC(=O)c2c(O)cc(O)cc2O1</smiles>

1<smiles>[R]Oc1ccc(C2CC(=O)c3c(O[R2])cc(O[R])cc3O2)cc1</smiles>

1a-10a

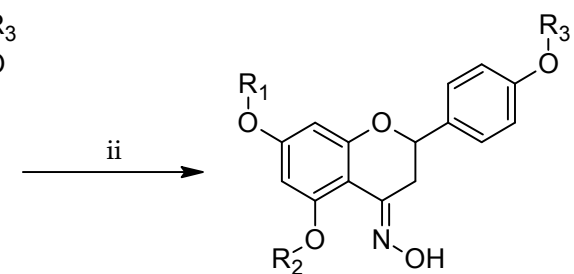

$1 b-10 b$

1a, $1 \mathbf{b}: \mathrm{R}_{1}=\mathrm{CH}_{3^{\prime}}, \mathrm{R}_{2}=\mathrm{H}, \mathrm{R}_{3}=\mathrm{H}$

2a, 2b: $\mathrm{R}_{1}=\mathrm{CH}_{3}, \mathrm{R}_{2}=\mathrm{H}, \mathrm{R}_{3}=\mathrm{CH}_{3}$

3a, 3b: $\mathrm{R}_{1}=\mathrm{CH}_{3}, \mathrm{R}_{2}=\mathrm{CH}_{3}, \mathrm{R}_{3}=\mathrm{CH}_{3}$

4a, $4 \mathrm{~b}: \mathrm{R}_{1}=\mathrm{CH}_{2} \mathrm{CH}_{3}, \mathrm{R}_{2}=\mathrm{H}, \mathrm{R}_{3}=\mathrm{H}$

5a, 5b: $\mathrm{R}_{1}=\mathrm{CH}_{2} \mathrm{CH}_{3^{\prime}} \mathrm{R}_{2}=\mathrm{H}, \mathrm{R}_{3}=\mathrm{CH}_{2} \mathrm{CH}_{3}$

6a, 6b: $\mathrm{R}_{1}=\mathrm{CH}_{2} \mathrm{CH}_{3}, \mathrm{R}_{2}=\mathrm{CH}_{2} \mathrm{CH}_{3^{\prime}} \mathrm{R}_{3}=\mathrm{CH}_{2} \mathrm{CH}_{3}$

$7 \mathrm{a}, 7 \mathbf{b}: \mathrm{R}_{1}=\mathrm{CH}_{2}\left(\mathrm{CH}_{2}\right)_{3} \mathrm{CH}_{3}, \mathrm{R}_{2}=\mathrm{H}, \mathrm{R}_{3}=\mathrm{H}$

8a, 8b: $\mathrm{R}_{1}=\mathrm{CH}_{2}\left(\mathrm{CH}_{2}\right)_{3} \mathrm{CH}_{3^{\prime}}, \mathrm{R}=\mathrm{H}, \mathrm{R}_{3}=\mathrm{CH}_{2}\left(\mathrm{CH}_{2}\right)_{3} \mathrm{CH}_{3}$

9a, 9b: $\mathrm{R}_{1}=\mathrm{CH}_{2}\left(\mathrm{CH}_{2}\right)_{10} \mathrm{CH}_{3}, \mathrm{R}_{2}=\mathrm{H}, \mathrm{R}_{3}=\mathrm{H}$

10a, 10b: $\mathrm{R}_{1}=\mathrm{CH}_{2}\left(\mathrm{CH}_{2}\right)_{10} \mathrm{CH}_{3^{\prime}} \mathrm{R}_{2}=\mathrm{H}, \mathrm{R}_{3}=\mathrm{CH}_{2}\left(\mathrm{CH}_{2}\right)_{10} \mathrm{CH}_{3}$

Scheme 1. Synthesis of $O$-alkyl derivatives $\mathbf{1 a}-\mathbf{1 0 a}$ and their oximes $\mathbf{1} \mathbf{b}-\mathbf{1 0 b}$; Reaction conditions: (i) alkyl iodide, $\left(\mathrm{CH}_{3}\right)_{2} \mathrm{CO}$ or DMF, $\mathrm{K}_{2} \mathrm{CO}_{3}$, r.t., $24-96 \mathrm{~h}$; (ii) $\mathrm{NH}_{2} \mathrm{OH} \cdot \mathrm{HCl}, \mathrm{CH}_{3} \mathrm{COONa}, \mathrm{EtOH}, 4{ }^{\circ} \mathrm{C}$, 24-96 h.

The structures of these compounds were confirmed by ${ }^{1} \mathrm{H}$ - and ${ }^{13} \mathrm{C}-\mathrm{NMR}$. Analysis of signals in ${ }^{1} \mathrm{H}-\mathrm{NMR}$ spectrum of $\mathrm{O}$-alkyl derivatives allowed to identify the methyl, ethyl, pentyl, and dodecyl groups attached to the 5, 7 and $4^{\prime}$ positions in naringenin (1a-10a). In the case of sakuranetin (7-O-methylnaringenin, 1a), signals at $12.02 \mathrm{ppm}$ attributed to the hydroxyl moiety attached to the C-5 position, and at $5.21 \mathrm{ppm}$ due to the substituent at $\mathrm{C}-4^{\prime}$ were observed. In the case of 7,4'-di-O-methylnaringenin (2a), only one singlet at $12.03 \mathrm{ppm}$ was observed, which confirmed the substitution of methyl groups at the $\mathrm{C}-4^{\prime}$ and $\mathrm{C}-7$ positions. The shift of the signal due to the hydroxyl moiety at C- 5 to the $12.03 \mathrm{ppm}$ is caused by the formation of intramolecular hydrogen bonding with the carbonyl group. Moreover, this bonding has an effect on the low reactivity of this group with alkyl iodide [24]. In view of the thermodynamic equilibrium between flavanones and chalcones, signals from $\mathrm{H}-2$ at $5.36 \mathrm{ppm}(\mathrm{dd}, J=13.2,3.0 \mathrm{~Hz}), \mathrm{H}-3_{\mathrm{a}}$ at $3.09 \mathrm{ppm}(\mathrm{dd}, J=17.2,13.2 \mathrm{~Hz})$ and $\mathrm{H}-3_{\mathrm{b}}$ at $2.79 \mathrm{ppm}$ $(\mathrm{dd}, J=17.2,3.0 \mathrm{~Hz})$ confirmed that the obtained derivatives had a flavanone skeleton. Furthermore, the signal at $196.23 \mathrm{ppm}$ in the ${ }^{13} \mathrm{C}-\mathrm{NMR}$ spectrum provides information about the presence of a carbonyl group in each product. In the case of oxime derivatives (1b-10b), a peak from the $=\mathrm{NOH}$ group at 11.03-10.89 ppm was observed. Besides, the downshift from $196.21 \mathrm{ppm}$ to $154.85 \mathrm{ppm}$ in the ${ }^{13} \mathrm{C}-\mathrm{NMR}$ spectra indicated the replacement of the carbonyl group with the oxime moiety.

In our study, the biological properties of the obtained derivatives were verified. These studies were performed to describe the inhibitory effect of the $O$-alkyl derivatives (1a-10a) (Table 1$)$ and their oximes (1)-10b) (Table 2) on two strains of bacteria and four strains of fungi. 
Table 1. Antimicrobial activity of $O$-alkyl derivatives of naringenin 1a-10a.

\begin{tabular}{|c|c|c|c|c|c|c|c|}
\hline & Strain & E. coli & S. aureus & C. albicans & A. alternata & F. linii & A. niger \\
\hline \multirow{2}{*}{ Control } & Lag-phase (h) & 4.0 & 2.5 & 3.0 & 16.5 & 14.5 & 11.0 \\
\hline & $\Delta \mathrm{OD}$ & 1.65 & 1.74 & 1.60 & 1.87 & 1.96 & 2.14 \\
\hline \multirow{2}{*}{ NG } & Lag-phase (h) & 15.0 & 4.5 & 5.0 & 20.0 & - & 5.5 \\
\hline & $\Delta \mathrm{OD}$ & 1.30 & 1.49 & 1.50 & 1.34 & 0 & 1.74 \\
\hline \multirow{2}{*}{$1 \mathrm{a}$} & Lag-phase (h) & 5.5 & 3.5 & 5.0 & 21.5 & 26.0 & 9.0 \\
\hline & $\Delta \mathrm{OD}$ & 0.75 & 1.59 & 1.45 & 0.72 & 1.17 & 1.55 \\
\hline \multirow{2}{*}{$2 a$} & Lag-phase (h) & 4.0 & 4.0 & 4.0 & 16.0 & 9.5 & 12.5 \\
\hline & $\Delta \mathrm{OD}$ & 0.63 & 1.73 & 1.09 & 0.49 & 0.72 & 1.49 \\
\hline \multirow{2}{*}{$3 a$} & Lag-phase (h) & 4.5 & 4.0 & 5.0 & 14.0 & 19.5 & 15.0 \\
\hline & $\Delta \mathrm{OD}$ & 0.53 & 1.73 & 1.57 & 1.31 & 1.81 & 1.21 \\
\hline \multirow[b]{2}{*}{$4 a$} & Lag-phase (h) & 4.0 & 2.5 & 5.5 & 25.0 & 26.5 & 6.5 \\
\hline & $\Delta \mathrm{OD}$ & 0.52 & 1.65 & 1.35 & 0.46 & 1.18 & 1.36 \\
\hline \multirow[b]{2}{*}{$5 a$} & Lag-phase (h) & 4.5 & 5.0 & 5.5 & 18.0 & 24.0 & 7.5 \\
\hline & $\Delta \mathrm{OD}$ & 0.51 & 1.64 & 0.93 & 0.48 & 0.88 & 1.03 \\
\hline \multirow{2}{*}{$6 a$} & Lag-phase (h) & 5.0 & 2.5 & 5.5 & 19.5 & 14.0 & 13.0 \\
\hline & $\Delta \mathrm{OD}$ & 0.24 & 1.07 & 0.50 & 0.47 & 0.52 & 0.54 \\
\hline \multirow{2}{*}{$7 a$} & Lag-phase (h) & 4.0 & 4.0 & 7.0 & 19.0 & 25.5 & 32.5 \\
\hline & $\Delta \mathrm{OD}$ & 0.51 & 1.67 & 1.19 & 1.00 & 1.44 & 1.00 \\
\hline \multirow{2}{*}{$8 a$} & Lag-phase (h) & - & 4.5 & 6.5 & 9.5 & 34.0 & - \\
\hline & $\Delta \mathrm{OD}$ & 0 & 1.46 & 1.00 & 0.47 & 0.33 & 0 \\
\hline \multirow{2}{*}{$9 a$} & Lag-phase (h) & - & 26.0 & 5.5 & 23.0 & 3.5 & 38.5 \\
\hline & $\Delta \mathrm{OD}$ & 0 & 0.83 & 0.97 & 0.42 & 0.98 & 0.96 \\
\hline \multirow{2}{*}{$10 a$} & Lag-phase (h) & 3.0 & 3.0 & 0.5 & 33.0 & - & 6.0 \\
\hline & $\Delta \mathrm{OD}$ & 0.23 & 0.91 & 1.19 & 1.63 & 0 & 1.58 \\
\hline
\end{tabular}

NG—naringenin; OD—Optical Density (OD was measured for $\lambda 560 \mathrm{~nm}$ ).

Table 2. Antimicrobial activity of oximes $\mathbf{1 b} \mathbf{- 1 0 b}$.

\begin{tabular}{|c|c|c|c|c|c|c|c|}
\hline & train & E. coli & S. aureus & C. albicans & A. alternata & F. linii & A. niger \\
\hline \multirow{2}{*}{ Control } & Lag-phase (h) & 4.0 & 2.5 & 3.0 & 16.5 & 14.5 & 11.0 \\
\hline & $\Delta \mathrm{OD}$ & 1.65 & 1.74 & 1.60 & 1.87 & 1.96 & 2.14 \\
\hline \multirow{2}{*}{ NG-OX } & Lag-phase (h) & - & 3.5 & 4.0 & 21.5 & 29.0 & - \\
\hline & $\Delta \mathrm{OD}$ & 0 & 1.66 & 1.69 & 0.96 & 1.20 & 0 \\
\hline \multirow{2}{*}{$1 b$} & Lag-phase (h) & 5.0 & 4.0 & 5.0 & 37.5 & 26.5 & 45.5 \\
\hline & $\Delta \mathrm{OD}$ & 0.73 & 1.46 & 1.30 & 0.69 & 1.41 & 0.49 \\
\hline \multirow{2}{*}{$2 b$} & Lag-phase (h) & 3.5 & 2.0 & 4.0 & 16.0 & 13.0 & 4.0 \\
\hline & $\Delta \mathrm{OD}$ & 0.82 & 1.46 & 0.68 & 0.77 & 0.62 & 0.59 \\
\hline \multirow{2}{*}{$3 b$} & Lag-phase (h) & 4.0 & 2.5 & 10.0 & 11.5 & 11.0 & 10.0 \\
\hline & $\Delta \mathrm{OD}$ & 0.74 & 1.90 & 1.29 & 1.10 & 1.51 & 1.10 \\
\hline \multirow[b]{2}{*}{$4 b$} & Lag-phase (h) & 4.0 & 2.5 & 3.0 & 16.0 & 12.0 & 11.0 \\
\hline & $\Delta \mathrm{OD}$ & 0.80 & 1.20 & 0.81 & 1.03 & 0.92 & 0.88 \\
\hline \multirow[b]{2}{*}{$5 b$} & Lag-phase (h) & 4.5 & 2.0 & 4.0 & 19.0 & 13.0 & 5.0 \\
\hline & $\Delta \mathrm{OD}$ & 0.29 & 0.95 & 0.41 & 0.51 & 0.55 & 0.40 \\
\hline \multirow{2}{*}{$6 b$} & Lag-phase (h) & 6.0 & 4.5 & 11.0 & 11.5 & 10.5 & 9.5 \\
\hline & $\Delta \mathrm{OD}$ & 0.30 & 1.29 & 1.30 & 0.69 & 1.47 & 0.76 \\
\hline \multirow{2}{*}{$7 \mathrm{~b}$} & Lag-phase (h) & 5.0 & 2.0 & 3.0 & 16.0 & 13.0 & 11.0 \\
\hline & $\Delta \mathrm{OD}$ & 0.45 & 0.97 & 0.77 & 1.02 & 0.82 & 0.83 \\
\hline \multirow{2}{*}{$8 b$} & Lag-phase (h) & - & 3.5 & 6.0 & 18.5 & 22.0 & 37.5 \\
\hline & $\Delta \mathrm{OD}$ & 0 & 1.27 & 0.87 & 1.24 & 0.53 & 1.05 \\
\hline \multirow[b]{2}{*}{$9 b$} & Lag-phase (h) & - & 4.5 & 3.0 & 34.5 & - & 10.5 \\
\hline & $\Delta \mathrm{OD}$ & 0 & 0.35 & 0.22 & 0.54 & 0 & 0.58 \\
\hline \multirow{2}{*}{$10 b$} & Lag-phase (h) & 0.5 & 1.0 & 1.0 & 31.5 & - & 7.0 \\
\hline & $\Delta \mathrm{OD}$ & 0.27 & 0.80 & 1.03 & 1.24 & 0 & 1.71 \\
\hline
\end{tabular}

NG-OX—naringenin oxime; OD—Optical Density (OD was measured for $\lambda 560 \mathrm{~nm}$ ). 
Susceptibility to the tested compounds was an individual feature of each strain. In the case of 7,4'-di-O-pentylnaringenin (8a) complete growth inhibition of E. coli ATCC10536 and A. niger DSM1957 was observed. Furthermore, compound 9a totally inhibited the growth of E. coli ATCC10536. In comparison to naringenin, 10a showed a 6 times stronger restriction of E. coli ATCC10536 growth and considerable reduction of the adaptive phase from 15 to $3 \mathrm{~h}$. Moreover, this compound showed full growth inhibition of $F$. linii KB-F1. Lee et al. reported that 7-O-butylnaringenin displayed antimicrobial activity against methicillin-resistant $S$. aureus (MRSA). The action of this derivative was compared with that of naturally occurring flavonoids-quercetin and naringenin —and has been described as Minimal Inhibitory Concentration (MIC) [12]. In our research, 9a, which has a dodecyl group attached to the C-7 position in naringenin, exhibited the best inhibitory effect against $S$. aureus DSM799 among all the tested $O$-alkyl derivatives (Figure 2). The results of our studies suggest that elongation of the alkyl chain improves the inhibitory effect on $S$. aureus strain. The only compound that limited the differentiation of $C$. albicans DSM1386 was 6a. Satisfying results were observed for A. alternata CBS1526 in the presence of compounds $2 \mathbf{a}, 4 \mathbf{a}-6 \mathbf{a}, 8 \mathbf{a}$ and $9 \mathbf{a}$.

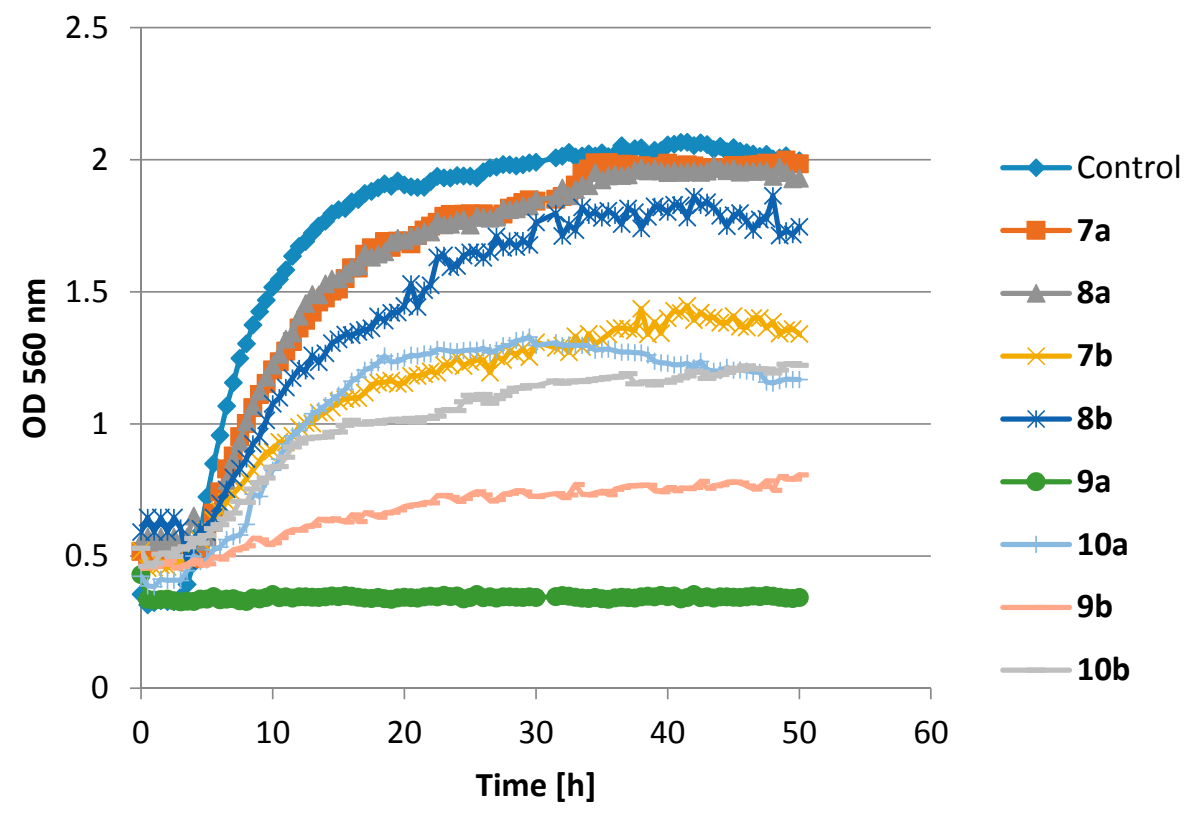

Figure 2. The effect of action of $O$-alkyl derivatives of naringenin (7a-10a) and their oximes (7b-10b) on the growth of S. aureus DSM799.

Biological assays performed on oxime derivatives allowed to evaluate the effect of introduction of the $=\mathrm{NOH}$ group on antimicrobial activity.

The strongest inhibitory effect was observed for compounds $\mathbf{8 b}$ and $\mathbf{9 b}$ in E. coli ATCC10536 culture. Moreover, naringenin oxime completely prevented the growth of this strain of bacteria and $A$. niger DSM1957. Compounds $\mathbf{2} \mathbf{b}$ and $\mathbf{5 b}$ also showed a strong inhibitory effect against this filamentous fungus. Derivative $\mathbf{9 b}$ also hindered A. niger DSM1957 growth and, additionally, prolonged the adaptive phase. Total inhibition of $F$. linii KB-F1 growth was achieved by the action of oximes $\mathbf{9 b}$ and 10b (Figure 3). 


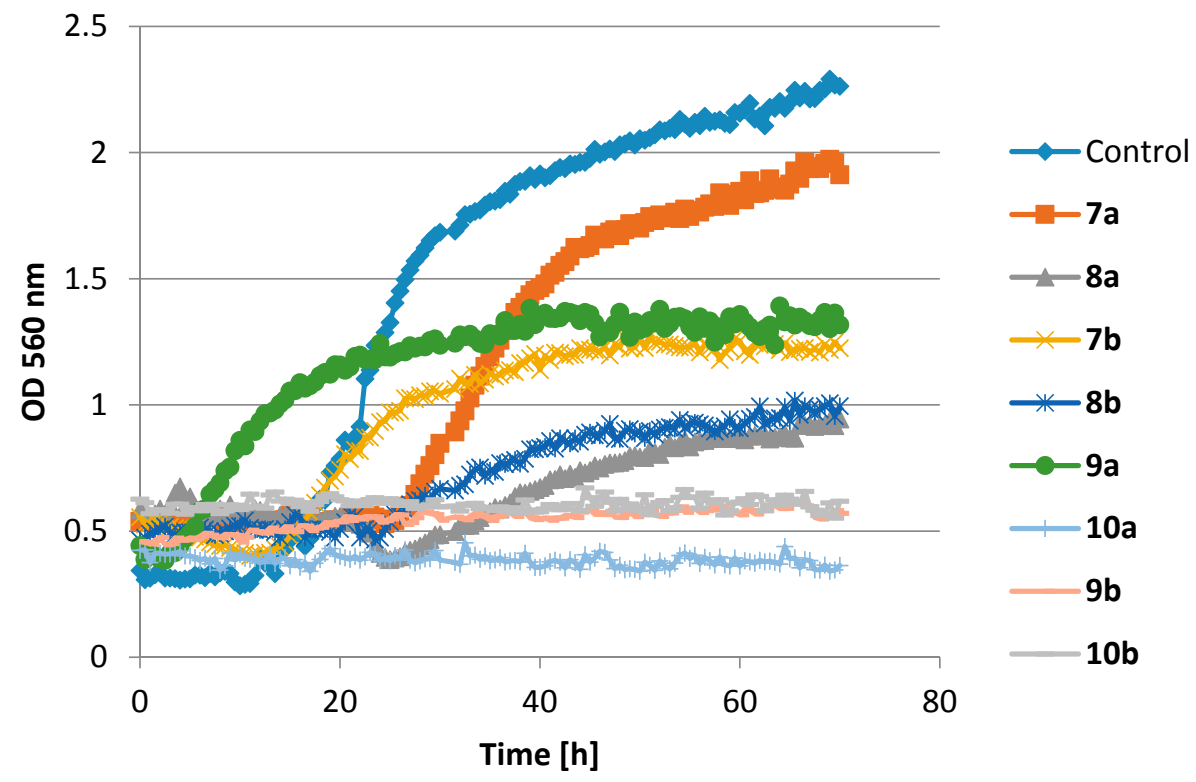

Figure 3. The effect of action of $O$-alkyl derivatives of naringenin $(\mathbf{7 a}-\mathbf{1 0 a})$ and their oximes $(\mathbf{7 b}-\mathbf{1 0 b})$ on the growth of F. linii KB-F1.

In the case of $S$. aureus DSM799, 7-O-dodecylnaringenin oxime $(\mathbf{9 b})$ had an inhibitory effect about 3 times stronger than 7-O-dodecylnaringenin (9a). Interestingly, the addition of $\mathbf{9 b}$ to the S. aureus DSM799 culture resulted in a shorter adaptive phase than that with 9a (Figure 2). Feng et al. described that oxime derivatives with long alkyl chains attached to 5,7-dihydroxy-4-chromanone show strong antimicrobial activity against $S$. aureus (MRSA). It is worth mentioning that the substitution of the $=\mathrm{NOH}$ group with a methyl or benzyl group is not favoured [25].

Yenjai et al. reported that extracts from Kaempferia parviflora contain various $O$-methyl derivatives of flavone. Furthermore, an investigation performed by a scientific group in Thailand showed that the introduction of the $=\mathrm{NOH}$ group instead of carbonyl enhances the biological properties of the modified compounds. The oxime derivative with two hydroxyl moieties at the C-5 and C-7 positions exhibited antifungal activity against $C$. albicans with an $\mathrm{IC}_{50}$ value of $48.98 \mu \mathrm{g} / \mathrm{mL}[22,26]$. A similar trend was observed in our study. In the case of $C$. albicans DSM1386, a stronger inhibitory effect of $\mathbf{9 b}$ than that of $9 \mathrm{a}$ was noticed. This proves that the introduction of the $=\mathrm{NOH}$ group significantly enhances the antimicrobial properties of this derivative.

Isosakuranetin (4'-O-methylnaringenin), obtained from flowers of Chromolaena odorata, exhibited moderate activity against Mycobacterium tuberculosis with the MIC value of $174.8 \mu \mathrm{M}$ [27]. Moreover, it decreased growth of Helicobacter pylori but hardly inhibited the urease activity of this strain of bacteria [28]. In our investigation, the isomer of isosakuranetin-sakuranetin (1a) exhibited a satisfactory inhibitory effect. This effect was about 2 times stronger than that of naringenin for $E$. coli ATCC10536 $(\triangle \mathrm{OD}=0.75)$ and A. alternata CBS1526 $(\triangle \mathrm{OD}=0.72)$ culture, but was not as strong as that of compounds $8 \mathbf{a}$ and $9 \mathrm{a}(\triangle \mathrm{OD}=0)$. Our studies confirmed that elongation of the hydrophobic chain increased the antimicrobial activities.

The therapeutic potential of oxime derivatives of flavonoids has not been well studied. Ilboudo et al. reported that the oxime obtained by chemical modification of the butanolic fraction from Mentha piperita exhibited stronger antifungal activity against Phoma sorghina and Fusarium moniliforme [29]. In our investigation, the replacement of carbonyl with the oxime group had a significant influence on not only F. linii KB-F1 $(9 \mathbf{a}-\Delta \mathrm{OD}=0.98, \mathbf{9 b}-\Delta \mathrm{OD}=0)$, but also $C$. albicans DSM1386 $(9 \mathbf{a}-\Delta \mathrm{OD}=0.97$, $\mathbf{9 b}-\Delta \mathrm{OD}=0.22)$ and $A$. niger DSM1957 growth $(\mathbf{9} \mathbf{a}-\Delta \mathrm{OD}=0.96, \mathbf{9 b}-\Delta \mathrm{OD}=0.58)$. 


\section{Materials and Methods}

\subsection{Chemicals}

Naringenin, iodomethane, iodoethane, 1-iodopentane and 1-iodododecane were purchased from Sigma-Aldrich Chemical Co. (Steinheim, Germany), hydroxylamine hydrochloride from LOBA Feinchemie $\mathrm{GmbH}$ (Fischamed, Austria), anhydrous sodium acetate and potassium carbonate from Chempur (Piekary Śląskie, Poland). Anhydrous solvents were prepared according to standard procedures. All organic solvents were of analytical grade.

\subsection{Analysis}

The reaction progress was analysed by thin layer chromatography (TLC) on silica gel-coated aluminium plates with fluorescent indicator (DC-Alufolien, Kieselgel $60 \mathrm{~F}_{254}$; Merck, Darmstadt, Germany). Products were detected by spraying the plates with a solution of $1 \% \mathrm{Ce}\left(\mathrm{SO}_{4}\right)_{2}$ and $2 \%$ $\mathrm{H}_{3}\left[\mathrm{P}\left(\mathrm{Mo}_{3} \mathrm{O}_{10}\right)_{4}\right]$ in $5 \% \mathrm{H}_{2} \mathrm{SO}_{4}$ and subsequently visualised by heating. Crude products were purified by liquid column chromatography using silica gel (Kieselgel 60, 230-400 mesh, Merck). The purity of the products was analysed by HPLC on a Waters 2690 (Milford, MA, USA) with Photodiode Array Detector Waters 996. The HPLC apparatus was equipped with a reverse-phase C-18 column (Phenomenex, Torrance, CA, United States, Kinetex 5u XB-C18 100A, $250 \mathrm{~mm} \times 4.6 \mathrm{~mm}$ ), which was thermostated at $28{ }^{\circ} \mathrm{C}$, and analysed samples were kept at $12{ }^{\circ} \mathrm{C}$. The mobile phase consisted of two eluents: $\mathrm{A}-1 \% \mathrm{HCOOH}$ in $\mathrm{MeCN}$ and $\mathrm{B}-1 \% \mathrm{HCOOH}$ in $\mathrm{H}_{2} \mathrm{O}$. Elution gradient was started from $55 \%$ of eluent A to $45 \%$ of eluent B over $21 \mathrm{~min}$. A flow rate of $1.5 \mathrm{~mL} / \mathrm{min}$ was used. The samples were dissolved in methanol.

Nuclear magnetic resonance (NMR) analysis was performed to elucidate the structure of the received compounds. ${ }^{1} \mathrm{H}-\mathrm{NMR}$ and ${ }^{13} \mathrm{C}-\mathrm{NMR}$ spectra were recorded on a Bruker Avance ${ }^{\mathrm{TM}} 600 \mathrm{MHz}$ spectrometer (Bruker, Billerica, MA, USA) with acetone-d6, chloroform-d, and dimethyl sulfoxide-d6 as solvents (Supplementary Materials, Figures S1-S40).

Positive-ion HR ESI-MS spectra were measured on a Bruker ESI-Q-TOF Maxis Impact Mass Spectrometer (Bruker, Billerica, MA, USA). The direct infusion of ESI-MS parameters: the mass spectrometer was operated in positive ion mode with the potential between the spray needle and the orifice $3.5 \mathrm{kV}$, nebulizer pressure of 0.4 bar, and a drying gas flow rate of $3.0 \mathrm{~L} / \mathrm{min}$ at $200{ }^{\circ} \mathrm{C}$. The sample flow rate was $3.0 \mu \mathrm{L} / \mathrm{min}$. Ionization mass spectra were collected at the ranges $\mathrm{m} / z$ 50-1250.

UV spectra were recorded in methanol on a Cintra 303 spectrophotometer (GBC, Braeside, Australia). Melting points (uncorrected) were determined on a Boetius apparatus (Jena, Germany).

\subsection{Synthesis of O-Alkyl Derivatives of Naringenin}

\subsubsection{Synthesis of Mono- (1a, $\mathbf{4 a}, \mathbf{7 a}, \mathbf{9 a})$ and Di-O-alkyl Derivatives of Naringenin (2a, 5a, 8a, 10a)}

Anhydrous potassium carbonate $(11.02 \mathrm{mmol})$ and the relevant alkyl iodide $(36.73 \mathrm{mmol})$ were added to naringenin $(7.35 \mathrm{mmol})$ dissolved in anhydrous acetone $(20 \mathrm{~mL})$. Reactions were performed for 24-96 $\mathrm{h}$ at room temperature. Then, the organic solvent was evaporated, and the resultant reaction mixture was treated with a saturated solution of sodium chloride $(40 \mathrm{~mL})$ and extracted with diethyl ether $(3 \times 50 \mathrm{~mL})$. The organic solvent was dried over sodium sulphate and concentrated on a vacuum evaporator. The crude products were separated by column chromatography.

\subsubsection{Synthesis of Tri-O-alkyl Derivatives of Naringenin (3a, 6a)}

Anhydrous potassium carbonate $(22.04 \mathrm{mmol})$ and the appropriate alkyl iodide (22.04 mmol) were added to naringenin $(3.67 \mathrm{mmol})$ dissolved in DMF $(10 \mathrm{~mL})$. Reactions were performed for 7-24 h at room temperature. Then, $1 \mathrm{M} \mathrm{HCl}$ was added dropwise until $\mathrm{pH} 7$ was reached. The resultant mixture was extracted with methylene chloride $(3 \times 50 \mathrm{~mL})$. The organic solvent was dried over 
sodium sulphate and concentrated on a vacuum evaporator. The crude products were isolated by column chromatography.

7-O-Methylnaringenin (1a), Yield 39.6\% (2.08 g), yellow powder, m.p. $144{ }^{\circ} \mathrm{C}$, lit. $143-144{ }^{\circ} \mathrm{C}[30]$. ${ }^{1} \mathrm{H}-\mathrm{NMR}\left(600 \mathrm{MHz}, \mathrm{CDCl}_{3}\right) \delta(\mathrm{ppm}): 12.02(\mathrm{~s}, 1 \mathrm{H}, \mathrm{OH}-5), 7.36-7.30\left(\mathrm{~m}, 2 \mathrm{H}, \mathrm{AA}^{\prime} \mathrm{BB}^{\prime}, \mathrm{H}-2^{\prime}, \mathrm{H}-6^{\prime}\right)$, 6.91-6.86 (m, 2H, AA'BB' $\left.{ }^{\prime}, \mathrm{H}-3^{\prime}, \mathrm{H}-5^{\prime}\right), 6.07(\mathrm{~d}, J=2.2 \mathrm{~Hz}, 1 \mathrm{H}, \mathrm{H}-6), 6.04(\mathrm{~d}, J=2.2 \mathrm{~Hz}, 1 \mathrm{H}, \mathrm{H}-8), 5.36$ $(\mathrm{dd}, J=13.2,3.0 \mathrm{~Hz}, 1 \mathrm{H}, \mathrm{H}-2), 5.21\left(\mathrm{~s}, 1 \mathrm{H}, \mathrm{OH}-4^{\prime}\right), 3.81\left(\mathrm{~s}, 3 \mathrm{H},-\mathrm{CH}_{3}\right), 3.09(\mathrm{dd}, J=17.2,13.2 \mathrm{~Hz}, 1 \mathrm{H}$, $\mathrm{H}-3_{\mathrm{a}}$ ), 2.79 (dd, $\left.J=17.2,3.0 \mathrm{~Hz}, 1 \mathrm{H}, \mathrm{H}-3_{\mathrm{b}}\right) .{ }^{13} \mathrm{C}-\mathrm{NMR}\left(150 \mathrm{MHz}, \mathrm{CDCl}_{3}\right) \delta(\mathrm{ppm}): 196.21(\mathrm{C}=\mathrm{O}), 168.15$, $164.25,163.02,156.28,130.67,128.11,115.82,103.27,95.26,94.41,79.11,55.85,43.34$. HR ESI-MS $m / z$ calculated for $\mathrm{C}_{16} \mathrm{H}_{14} \mathrm{O}_{5}[\mathrm{M}+\mathrm{H}]^{+} 287.0914$, found $[\mathrm{M}+\mathrm{H}]^{+}$287.0917, lit. HR ESI-MS $m / z$ calculated for $\mathrm{C}_{16} \mathrm{H}_{13} \mathrm{O}_{5}[\mathrm{M}-\mathrm{H}]^{-}$285.0763, found $[\mathrm{M}-\mathrm{H}]^{-} 285.0771$ [8].

7,4'-Di-O-methylnaringenin (2a), Yield 42.3\% (2.33 g), pale yellow powder, m.p. $112-115^{\circ} \mathrm{C}$, lit. $114-115$ ${ }^{\circ} \mathrm{C}[31] .{ }^{1} \mathrm{H}-\mathrm{NMR}\left(600 \mathrm{MHz}, \mathrm{CDCl}_{3}\right) \delta(\mathrm{ppm}): 12.03(\mathrm{~s}, 1 \mathrm{H}, \mathrm{OH}-5), 7.41-7.35\left(\mathrm{~m}, 2 \mathrm{H}, \mathrm{AA}^{\prime} \mathrm{BB}^{\prime}, \mathrm{H}-2^{\prime}, \mathrm{H}-6^{\prime}\right)$, 6.98-6.91 (m, 2H, AA'BB' ${ }^{\prime}$ H-3', $\left.\mathrm{H}-5^{\prime}\right), 6.07$ (d, J = 2.3 Hz, 1H, H-6), 6.04 (d, J = 2.3 Hz, 1H, H-8), 5.36 (dd, $J=13.1,3.0 \mathrm{~Hz}, 1 \mathrm{H}, \mathrm{H}-2), 3.83\left(\mathrm{~s}, 3 \mathrm{H},-\mathrm{CH}_{3}\right), 3.80\left(\mathrm{~s}, 3 \mathrm{H},-\mathrm{CH}_{3}\right), 3.10\left(\mathrm{dd}, J=17.2,13.1 \mathrm{~Hz}, 1 \mathrm{H}, \mathrm{H}-3_{\mathrm{a}}\right)$, $2.79\left(\mathrm{dd}, J=17.2,3.0 \mathrm{~Hz}, 1 \mathrm{H}, \mathrm{H}-3_{\mathrm{b}}\right) .{ }^{13} \mathrm{C}-\mathrm{NMR}\left(150 \mathrm{MHz}, \mathrm{CDCl}_{3}\right) \delta(\mathrm{ppm}): 196.16(\mathrm{C}=\mathrm{O}), 168.09,164.26$, $163.03,160.18,130.51,127.87,114.36,103.27,95.22,94.36,79.15,55.82,55.51,43.34$. HR ESI-MS $m / z$ calculated for $\mathrm{C}_{17} \mathrm{H}_{16} \mathrm{O}_{5}[\mathrm{M}+\mathrm{H}]^{+} 301.1071$, found $[\mathrm{M}+\mathrm{H}]^{+} 301.1086$, lit. HR ESI-MS $m / z$ calculated for $\mathrm{C}_{17} \mathrm{H}_{16} \mathrm{O}_{5} \mathrm{Na}^{+}[\mathrm{M}+\mathrm{Na}]^{+} 323.0890$, found $[\mathrm{M}+\mathrm{Na}]^{+} 323.0873$ [31].

5,7,4'-Tri-O-methylnaringenin (3a), Yield $72.3 \%\left(0.840\right.$ g), white powder, m.p. $126-129{ }^{\circ} \mathrm{C}$, lit. $120-122$ ${ }^{\circ} \mathrm{C}$ [22]. ${ }^{1} \mathrm{H}-\mathrm{NMR}\left(600 \mathrm{MHz}, \mathrm{CDCl}_{3}\right) \delta(\mathrm{ppm}): 7.41-7.35$ (m, 2H, $\left.\mathrm{AA}^{\prime} \mathrm{BB}^{\prime}, \mathrm{H}-2^{\prime}, \mathrm{H}-6^{\prime}\right), 6.97-6.91(\mathrm{~m}, 2 \mathrm{H}$, $\left.\mathrm{AA}^{\prime} \mathrm{BB}^{\prime}, \mathrm{H}-3^{\prime} \mathrm{H}-5^{\prime}\right), 6.14$ (d, $\left.J=2.3 \mathrm{~Hz}, 1 \mathrm{H}, \mathrm{H}-6\right), 6.09(\mathrm{~d}, J=2.3 \mathrm{~Hz}, 1 \mathrm{H}, \mathrm{H}-8), 5.35$ (dd, $J=13.2,2.9$ $\mathrm{Hz}, 1 \mathrm{H}, \mathrm{H}-2), 3.89\left(\mathrm{~s}, 3 \mathrm{H},-\mathrm{CH}_{3}\right), 3.83\left(\mathrm{~s}, 3 \mathrm{H},-\mathrm{CH}_{3}\right), 3.81\left(\mathrm{~s}, 3 \mathrm{H},-\mathrm{CH}_{3}\right), 3.03(\mathrm{dd}, J=16.5,2.9 \mathrm{~Hz}, 1 \mathrm{H}$, $\left.\mathrm{H}-3_{\mathrm{a}}\right), 2.76\left(\mathrm{dd}, J=16.5,2.9 \mathrm{~Hz}, 1 \mathrm{H}, \mathrm{H}-3_{\mathrm{b}}\right) .{ }^{13} \mathrm{C}-\mathrm{NMR}\left(150 \mathrm{MHz}, \mathrm{CDCl}_{3}\right) \delta(\mathrm{ppm}): 189.59(\mathrm{C}=\mathrm{O}), 166.06$, 165.20, 162.40, 160.03, 130.92, 127.84, 114.27, 106.12, 93.66, 93.25, 79.12, 56.30, 55.72, 55.49, 45.54. HR ESI-MS $m / z$ calculated for $\mathrm{C}_{18} \mathrm{H}_{18} \mathrm{O}_{5}[\mathrm{M}+\mathrm{H}]^{+} 315.1227$, found $[\mathrm{M}+\mathrm{H}]^{+} 315.1229$, lit. HR ESI-MS $m / z$ calculated for $\mathrm{C}_{18} \mathrm{H}_{18} \mathrm{O}_{5} \mathrm{Na}^{+}[\mathrm{M}+\mathrm{Na}]^{+}$337.1052, found [M + Na] 337.1052 [22].

7-O-Ethylnaringenin (4a), Yield 67.8\% (1.50 g), yellow powder, m.p. $132-134{ }^{\circ} \mathrm{C}$, lit. $130-131{ }^{\circ} \mathrm{C}$ [32]. ${ }^{1} \mathrm{H}-\mathrm{NMR}\left(600 \mathrm{MHz}, \mathrm{CDCl}_{3}\right) \delta(\mathrm{ppm}): 12.01(\mathrm{~s}, 1 \mathrm{H}, \mathrm{OH}-5), 7.32\left(\mathrm{~d}, J=8.1 \mathrm{~Hz}, 2 \mathrm{H}, \mathrm{H}-2^{\prime}, \mathrm{H}-6^{\prime}\right), 6.88(\mathrm{~d}$, $\left.J=8.1 \mathrm{~Hz}, 2 \mathrm{H}, \mathrm{H}-3^{\prime}, \mathrm{H}^{\prime} 5^{\prime}\right), 6.06(\mathrm{~d}, J=2.3 \mathrm{~Hz}, 1 \mathrm{H}, \mathrm{H}-6), 6.03(\mathrm{~d}, J=2.3 \mathrm{~Hz}, 1 \mathrm{H}, \mathrm{H}-8), 5.58\left(\mathrm{~s}, 1 \mathrm{H}, \mathrm{OH}-4^{\prime}\right)$, $5.34(\mathrm{dd}, J=12.9,3.0 \mathrm{~Hz}, 1 \mathrm{H}, \mathrm{H}-2), 4.03\left(\mathrm{q}, J=7.0 \mathrm{~Hz}, 2 \mathrm{H},-\mathrm{CH}_{2}-\right), 3.09\left(\mathrm{dd}, J=17.2,12.9 \mathrm{~Hz}, 1 \mathrm{H}, \mathrm{H}-3_{\mathrm{a}}\right)$, $2.78\left(\mathrm{dd}, J=17.2,3.0 \mathrm{~Hz}, 1 \mathrm{H}, \mathrm{H}-3_{\mathrm{b}}\right), 1.40\left(\mathrm{t}, J=7.0 \mathrm{~Hz}, 3 \mathrm{H},-\mathrm{CH}_{3}\right) .{ }^{13} \mathrm{C}-\mathrm{NMR}\left(150 \mathrm{MHz}, \mathrm{CDCl}_{3}\right) \delta(\mathrm{ppm})$ : 196.17 (C=O), 167.61, 164.21, 163.03, 156.26, 130.72, 128.11, 115.81, 103.17, 95.69, 94.76, 79.06, 64.26, 43.31, 14.67. HR ESI-MS $m / z$ calculated for $\mathrm{C}_{17} \mathrm{H}_{16} \mathrm{O}_{5}[\mathrm{M}+\mathrm{H}]^{+} 301.1071$, found $[\mathrm{M}+\mathrm{H}]^{+} 301.1081$.

7,4'-Di-O-ethylnaringenin (5a), Yield 23.5\% (0.566 g), white powder, m.p. 97-102 ${ }^{\circ} \mathrm{C}$, lit. $97-98^{\circ} \mathrm{C}$ [32]. ${ }^{1} \mathrm{H}-\mathrm{NMR}\left(600 \mathrm{MHz}, \mathrm{CDCl}_{3}\right) \delta(\mathrm{ppm}): 12.02(\mathrm{~s}, 1 \mathrm{H}, \mathrm{OH}-5), 7.39-7.33\left(\mathrm{~m}, 2 \mathrm{H}, \mathrm{AA}^{\prime} \mathrm{BB}^{\prime}, \mathrm{H}-2^{\prime}, \mathrm{H}-6^{\prime}\right)$,

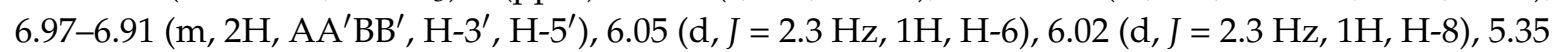
$(\mathrm{dd}, J=13.0,3.0 \mathrm{~Hz}, 1 \mathrm{H}, \mathrm{H}-2), 4.06\left(\mathrm{q}, J=7.0 \mathrm{~Hz}, 2 \mathrm{H},-\mathrm{CH}_{2}-\right), 4.03\left(\mathrm{q}, J=7.0 \mathrm{~Hz}, 2 \mathrm{H},-\mathrm{CH}_{2}-\right), 3.09(\mathrm{dd}$, $\left.J=17.1,13.0 \mathrm{~Hz}, 1 \mathrm{H}, \mathrm{H}-3_{\mathrm{a}}\right), 2.78\left(\mathrm{dd}, J=17.1,3.0 \mathrm{~Hz}, 1 \mathrm{H}, \mathrm{H}-3_{\mathrm{b}}\right), 1.43\left(\mathrm{t}, J=7.0 \mathrm{~Hz}, 3 \mathrm{H},-\mathrm{CH}_{3}\right), 1.40$ $\left(\mathrm{t}, J=7.0 \mathrm{~Hz}, 3 \mathrm{H},-\mathrm{CH}_{3}\right) .{ }^{13} \mathrm{C}-\mathrm{NMR}\left(150 \mathrm{MHz}, \mathrm{CDCl}_{3}\right) \delta[\mathrm{ppm}]: 196.12(\mathrm{C}=\mathrm{O}), 167.51,164.23,163.04$, $159.55,130.38,127.85,114.88,103.18,95.63,94.69,79.15,64.21,63.71,43.34,14.93,14.67$. HR ESI-MS $m / z$ calculated for $\mathrm{C}_{19} \mathrm{H}_{20} \mathrm{O}_{5}[\mathrm{M}+\mathrm{H}]^{+} 329.1386$, found $[\mathrm{M}+\mathrm{H}]^{+} 329.1400$.

5,7,4'-Tri-O-ethylnaringenin (6a), Yield 58.9\% (0.385 g), white powder, m.p. $117-120{ }^{\circ} \mathrm{C} .{ }^{1} \mathrm{H}-\mathrm{NMR}(600$ $\mathrm{MHz}, \mathrm{CDCl}_{3}$ ) $\delta$ (ppm): 7.39-7.34 (m, 2H, $\left.\mathrm{AA}^{\prime} \mathrm{BB}^{\prime}, \mathrm{H}-2^{\prime}, \mathrm{H}^{\prime} 6^{\prime}\right), 6.95-6.89\left(\mathrm{~m}, 2 \mathrm{H}, \mathrm{AA}^{\prime} \mathrm{BB}^{\prime}, \mathrm{H}-3^{\prime}, \mathrm{H}^{\prime} 5^{\prime}\right)$, $6.10(\mathrm{~d}, J=2.2 \mathrm{~Hz}, 1 \mathrm{H}, \mathrm{H}-6), 6.06$ (d, $J=2.2 \mathrm{~Hz}, 1 \mathrm{H}, \mathrm{H}-8), 5.33$ (dd, $J=13.4,2.9 \mathrm{~Hz}, 1 \mathrm{H}, \mathrm{H}-2), 4.11-4.07$ $\left(\mathrm{m}, 2 \mathrm{H},-\mathrm{CH}_{2}-\right), 4.07-4.00\left(\mathrm{~m}, 4 \mathrm{H}, 2 \mathrm{x}-\mathrm{CH}_{2}-\right), 3.02(\mathrm{dd}, J=16.5,13.4 \mathrm{~Hz}, 1 \mathrm{H}, \mathrm{H}-3 \mathrm{a}), 2.74(\mathrm{dd}, J=16.5,2.9$ 
$\mathrm{Hz}, 1 \mathrm{H}, \mathrm{H}-3 \mathrm{~b}), 1.51\left(\mathrm{t}, J=7.0 \mathrm{~Hz}, 3 \mathrm{H},-\mathrm{CH}_{3}\right), 1.42\left(\mathrm{t}, J=7.0 \mathrm{~Hz}, 3 \mathrm{H},-\mathrm{CH}_{3}\right) 1.41\left(\mathrm{t}, J=7.0 \mathrm{~Hz}, 3 \mathrm{H},-\mathrm{CH}_{3}\right)$. ${ }^{13} \mathrm{C}-\mathrm{NMR}\left(150 \mathrm{MHz}, \mathrm{CDCl}_{3}\right) \delta$ (ppm): 189.48 (C=O), 165.33, 165.14, 161.72, 159.38, 130.86, 127.82, 114.80, $106.15,94.38,93.93,79.13,64.74,64.01,63.68,45.66,14.93,14.72,14.69$. HR ESI-MS $m / z$ calculated for $\mathrm{C}_{21} \mathrm{H}_{24} \mathrm{O}_{5}[\mathrm{M}+\mathrm{H}]^{+}$357.1697, found [M + H] 357.1699 .

7-O-Pentylnaringenin (7a), Yield 72.3\% (1.81 g), yellow powder, m.p. $110-113{ }^{\circ} \mathrm{C} .{ }^{1} \mathrm{H}-\mathrm{NMR}(600 \mathrm{MHz}$, $\left.\mathrm{CDCl}_{3}\right) \delta(\mathrm{ppm}): 12.01(\mathrm{~s}, 1 \mathrm{H}, \mathrm{OH}-5), 7.35-7.31\left(\mathrm{~m}, 2 \mathrm{H}, \mathrm{AA}^{\prime} \mathrm{BB}^{\prime}, \mathrm{H}^{\prime} 2^{\prime}, \mathrm{H}-6^{\prime}\right), 6.91-6.85\left(\mathrm{~m}, 2 \mathrm{H}, \mathrm{AA}^{\prime} \mathrm{BB}^{\prime}\right.$, H-3', $\left.\mathrm{H}-5^{\prime}\right), 6.09$ (d, $\left.J=2.4 \mathrm{~Hz}, 1 \mathrm{H}, \mathrm{H}-6\right), 6.07$ (d, $\left.J=2.4 \mathrm{~Hz}, 1 \mathrm{H}, \mathrm{H}-8\right), 5.35$ (dd, $\left.J=13.0,3.0 \mathrm{~Hz}, 1 \mathrm{H}, \mathrm{H}-2\right)$, $5.19\left(\mathrm{~s}, 1 \mathrm{H}, \mathrm{OH}-4^{\prime}\right), 3.96\left(\mathrm{t}, J=6.6 \mathrm{~Hz}, 2 \mathrm{H},-\mathrm{CH}_{2}-\right), 3.08(\mathrm{dd}, J=17.1,13.0 \mathrm{~Hz}, 1 \mathrm{H}, \mathrm{H}-3 \mathrm{a}), 2.78(\mathrm{dd}, J=17.1$, $\left.3.0 \mathrm{~Hz}, 1 \mathrm{H}, \mathrm{H}-3_{\mathrm{b}}\right), 1.77\left(\mathrm{p}, J=6.9 \mathrm{~Hz}, 2 \mathrm{H},-\mathrm{CH}_{2}-\right), 1.44-1.33\left(\mathrm{~m}, 4 \mathrm{H}, 2 \mathrm{x}-\mathrm{CH}_{2}-\right), 0.92(\mathrm{t}, J=7.1 \mathrm{~Hz}, 3 \mathrm{H}$, $\left.-\mathrm{CH}_{3}\right) .{ }^{13} \mathrm{C}-\mathrm{NMR}\left(150 \mathrm{MHz}, \mathrm{CDCl}_{3}\right) \delta$ (ppm): 196.12 (C=O), 167.81, 164.21, 163.01, 156.24, 130.77, 128.10, $115.81,103.14,95.72,94.76,79.05,68.72,43.33,28.73,28.17,22.50,14.12$. HR ESI-MS $m / z$ calculated for $\mathrm{C}_{20} \mathrm{H}_{22} \mathrm{O}_{5}[\mathrm{M}+\mathrm{H}]^{+} 343.1540$, found $[\mathrm{M}+\mathrm{H}]^{+} 343.1547$.

7,4'-Di-O-pentylnaringenin (8a), Yield 30.8\% (0.932 g), pale yellow powder, m.p. $67-70{ }^{\circ} \mathrm{C} .{ }^{1} \mathrm{H}-\mathrm{NMR}$ $\left(600 \mathrm{MHz}, \mathrm{CDCl}_{3}\right) \delta(\mathrm{ppm}): 12.02$ (s, 1H, OH-5), 7.38-7.33 (m, 2H, $\left.\mathrm{AA}^{\prime} \mathrm{BB}^{\prime}, \mathrm{H}-2^{\prime}, \mathrm{H}-6^{\prime}\right), 6.96-6.92(\mathrm{~m}$, 2H, $\left.\mathrm{AA}^{\prime} \mathrm{BB}^{\prime}, \mathrm{H}-3^{\prime}, \mathrm{H}-5^{\prime}\right), 6.05(\mathrm{~d}, J=2.2 \mathrm{~Hz}, 1 \mathrm{H}, \mathrm{H}-6), 6.02(\mathrm{~d}, J=2.2 \mathrm{~Hz}, 1 \mathrm{H}, \mathrm{H}-8), 5.35(\mathrm{dd}, J=13.0,3.0$ $\mathrm{Hz}, 1 \mathrm{H}, \mathrm{H}-2), 3.97\left(\mathrm{t}, J=6.6 \mathrm{~Hz}, 2 \mathrm{H},-\mathrm{CH}_{2}-\right), 3.95\left(\mathrm{t}, J=6.6 \mathrm{~Hz}, 2 \mathrm{H},-\mathrm{CH}_{2}-\right), 3.09(\mathrm{dd}, J=17.2,13.0 \mathrm{~Hz}, 1 \mathrm{H}$, $\left.\mathrm{H}-3_{\mathrm{a}}\right), 2.77\left(\mathrm{dd}, J=17.2,3.0 \mathrm{~Hz}, 1 \mathrm{H}, \mathrm{H}-3_{\mathrm{b}}\right), 1.84-1.73\left(\mathrm{~m}, 4 \mathrm{H}, 2 \mathrm{x}-\mathrm{CH}_{2}-\right), 1.49-1.32\left(\mathrm{~m}, 8 \mathrm{H}, 4 \mathrm{x}-\mathrm{CH}_{2}-\right), 0.94$ $\left(\mathrm{t}, J=7.4 \mathrm{~Hz}, 3 \mathrm{H},-\mathrm{CH}_{3}\right), 0.92\left(\mathrm{t}, J=7.4 \mathrm{~Hz}, 3 \mathrm{H},-\mathrm{CH}_{3}\right) .{ }^{13} \mathrm{C}-\mathrm{NMR}\left(150 \mathrm{MHz}, \mathrm{CDCl}_{3}\right) \delta(\mathrm{ppm}): 196.10$ $(\mathrm{C}=\mathrm{O}), 167.72,164.22,163.05,159.75,130.33,127.82,114.89,103.15,95.66,94.70,79.14,68.68,68.26,43.34$, $29.05,28.73,28.32,28.17,22.59,22.51,14.16,14.12$. HR ESI-MS $m / z$ calculated for $\mathrm{C}_{25} \mathrm{H}_{32} \mathrm{O}_{5}[\mathrm{M}+\mathrm{H}]^{+}$ 413.2323, found $[\mathrm{M}+\mathrm{H}]^{+} 413.2345$.

7-O-Dodecylnaringenin (9a), Yield 70.2\% (1.14 g), pale yellow powder, m.p. $101-105{ }^{\circ} \mathrm{C} .{ }^{1} \mathrm{H}-\mathrm{NMR}$ (600 MHz, $\left.\mathrm{CDCl}_{3}\right) \delta(\mathrm{ppm}): 12.01(\mathrm{~s}, 1 \mathrm{H}, \mathrm{OH}-5), 7.36-7.31$ (m, 2H, $\left.\mathrm{AA}^{\prime} \mathrm{BB}^{\prime}, \mathrm{H}-2^{\prime}, \mathrm{H}-6^{\prime}\right), 6.91-6.85$ (m, $\left.2 \mathrm{H}, \mathrm{AA}^{\prime} \mathrm{BB}^{\prime}, \mathrm{H}-3^{\prime}, \mathrm{H}-5^{\prime}\right), 6.05(\mathrm{~d}, J=2.2 \mathrm{~Hz}, 1 \mathrm{H}, \mathrm{H}-6), 6.03(\mathrm{~d}, J=2.2 \mathrm{~Hz}, 1 \mathrm{H}, \mathrm{H}-8), 5.35(\mathrm{dd}, J=13.0,3.0$ $\mathrm{Hz}, 1 \mathrm{H}, \mathrm{H}-2), 5.00\left(\mathrm{~s}, 1 \mathrm{H}, \mathrm{OH}-4^{\prime}\right), 3.95\left(\mathrm{t}, J=6.6 \mathrm{~Hz}, 2 \mathrm{H},-\mathrm{CH}_{2}-\right), 3.08(\mathrm{dd}, J=17.1,13.0 \mathrm{~Hz}, 1 \mathrm{H}, \mathrm{H}-3 \mathrm{a})$, $2.78\left(\mathrm{dd}, J=17.1,3.0 \mathrm{~Hz}, 1 \mathrm{H}, \mathrm{H}-3_{\mathrm{b}}\right), 1.76\left(\mathrm{p}, J=6.6 \mathrm{~Hz}, 2 \mathrm{H},-\mathrm{CH}_{2}-\right), 1.44-1.38\left(\mathrm{~m}, 2 \mathrm{H},-\mathrm{CH}_{2}-\right), 1.34-1.20$ $\left(\mathrm{m}, 16 \mathrm{H}, 8 \mathrm{x}-\mathrm{CH}_{2}-\right), 0.88\left(\mathrm{t}, J=7.0 \mathrm{~Hz}, 3 \mathrm{H},-\mathrm{CH}_{3}\right) .{ }^{13} \mathrm{C}-\mathrm{NMR}\left(150 \mathrm{MHz}, \mathrm{CDCl}_{3}\right) \delta(\mathrm{ppm}): 196.04(\mathrm{C}=\mathrm{O})$, $167.77,164.22,162.98,156.20,130.83,128.10,115.80,103.14,95.71,94.74,79.05,68.73,43.37,32.06,29.79$, $29.77,29.72,29.67,29.49,29.43,29.03,26.03,22.84,14.28$. HR ESI-MS $m / z$ calculated for $\mathrm{C}_{27} \mathrm{H}_{36} \mathrm{O}_{5}$ $[\mathrm{M}+\mathrm{H}]^{+} 441.2636$, found $[\mathrm{M}+\mathrm{H}]^{+} 441.2654$.

7,4'-Di-O-dodecylnaringenin (10a), Yield 20.5\% (0.457 g), white powder, m.p. $60-62{ }^{\circ} \mathrm{C} .{ }^{1} \mathrm{H}-\mathrm{NMR}$ $\left(600 \mathrm{MHz}, \mathrm{CDCl}_{3}\right) \delta$ (ppm): 12.02 (s, 1H, OH-5), 7.38-7.34 (m, 2H, $\left.\mathrm{AA}^{\prime} \mathrm{BB}^{\prime}, \mathrm{H}-2^{\prime}, \mathrm{H}-6^{\prime}\right), 6.96-6.91$ (m, 2H, $\left.\mathrm{AA}^{\prime} \mathrm{BB}^{\prime}, \mathrm{H}-3^{\prime}, \mathrm{H}-5^{\prime}\right), 6.05(\mathrm{~d}, J=2.2 \mathrm{~Hz}, 1 \mathrm{H}, \mathrm{H}-6), 6.02(\mathrm{~d}, J=2.2 \mathrm{~Hz}, 1 \mathrm{H}, \mathrm{H}-8), 5.35(\mathrm{dd}, J=13.0,3.0$ $\mathrm{Hz}, 1 \mathrm{H}, \mathrm{H}-2), 3.97\left(\mathrm{t}, J=6.6 \mathrm{~Hz}, 2 \mathrm{H},-\mathrm{CH}_{2}-\right), 3.95\left(\mathrm{t}, J=6.6 \mathrm{~Hz}, 2 \mathrm{H},-\mathrm{CH}_{2}-\right), 3.09(\mathrm{dd}, J=17.2,13.0 \mathrm{~Hz}$, $\left.1 \mathrm{H}, \mathrm{H}-3_{\mathrm{a}}\right), 2.78\left(\mathrm{dd}, J=17.2,3.0 \mathrm{~Hz}, 1 \mathrm{H}, \mathrm{H}-3_{\mathrm{b}}\right), 1.83-1.71\left(\mathrm{~m}, 4 \mathrm{H}, 2 \mathrm{x}-\mathrm{CH}_{2}-\right), 1.48-1.38\left(\mathrm{~m}, 4 \mathrm{H}, 2 \mathrm{x}-\mathrm{CH}_{2}-\right)$, 1.34-1.21 (m, 32H, 16x- $\left.\mathrm{CH}_{2}-\right), 0.88\left(\mathrm{t}, J=7.0 \mathrm{~Hz}, 3 \mathrm{H},-\mathrm{CH}_{3}\right), 0.88\left(\mathrm{t}, J=7.0 \mathrm{~Hz}, 3 \mathrm{H},-\mathrm{CH}_{3}\right) .{ }^{13} \mathrm{C}-\mathrm{NMR}(150$ $\mathrm{MHz}, \mathrm{CDCl}_{3}$ ) $\delta$ (ppm): 196.12 (C=O), 167.73, 164.22, 163.04, 159.76, 130.32, 127.83, 114.90, 103.15, 95.66, $94.71,79.16,68.71,68.28,43.35,32.07,29.81,29.79,29.77,29.75,29.73,29.72,29.68,29.54,29.50,29.43$, 29.36, 29.04, 26.18, 26.03, 22.84, 14.28. HR ESI-MS $m / z$ calculated for $\mathrm{C}_{39} \mathrm{H}_{60} \mathrm{O}_{5}[\mathrm{M}+\mathrm{H}]^{+} 609.4514$, found $[\mathrm{M}+\mathrm{H}]^{+} 609.4499$.

\subsection{Synthesis of Oximes $(\mathbf{1} \mathbf{b}-\mathbf{1 0 b})$}

Hydroxylamine hydrochloride $(1.60 \mathrm{mmol})$ and anhydrous sodium acetate $(1.60 \mathrm{mmol})$ were added to the $O$-alkyl derivative of naringenin $(1.06 \mathrm{mmol})(\mathbf{1 a}-\mathbf{1 0 a})$ dissolved in anhydrous ethanol $(10 \mathrm{~mL})$. Reaction was performed on magnetic stirrer at $40-50{ }^{\circ} \mathrm{C}$. Then, the mixture was poured into ice water and the precipitated crystals were collected. The crude products were purified by column 
chromatography. In some cases, washing with cold water was sufficient to obtain the desired product with satisfactory purity.

7-O-Methylnaringenin oxime (1b), Yield 99.4\% (0.525 g), white powder, m.p. $195-200{ }^{\circ} \mathrm{C} .{ }^{1} \mathrm{H}-\mathrm{NMR}$ $\left(600 \mathrm{MHz}\right.$, acetone- $\left.d_{6}\right) \delta(\mathrm{ppm}): 11.03(\mathrm{~s}, 1 \mathrm{H}, \mathrm{NOH}), 10.40(\mathrm{~s}, 1 \mathrm{H}, \mathrm{OH}-5), 8.48\left(\mathrm{~s}, 1 \mathrm{H}, \mathrm{OH}-4^{\prime}\right), 7.42-7.34$ (m, 2H, AA' $\left.\mathrm{BB}^{\prime}, \mathrm{H}-2^{\prime}, \mathrm{H}-6^{\prime}\right), 6.94-6.86\left(\mathrm{~m}, 2 \mathrm{H}, \mathrm{AA}^{\prime} \mathrm{BB}^{\prime}, \mathrm{H}^{\prime} 3^{\prime}, \mathrm{H}-5^{\prime}\right), 6.05$ (d, J = 2.5 Hz, 1H, H-6), 6.04 $(\mathrm{d}, J=2.5 \mathrm{~Hz}, 1 \mathrm{H}, \mathrm{H}-8), 5.08(\mathrm{dd}, J=12.0,3.1 \mathrm{~Hz}, 1 \mathrm{H}, \mathrm{H}-2), 3.76\left(\mathrm{~s}, 3 \mathrm{H},-\mathrm{CH}_{3}\right), 3.46(\mathrm{dd}, J=17.1,3.1$ $\left.\mathrm{Hz}, 1 \mathrm{H}, \mathrm{H}-3_{\mathrm{a}}\right), 2.79\left(\mathrm{dd}, J=17.1,12.0 \mathrm{~Hz}, 1 \mathrm{H}, \mathrm{H}-3_{\mathrm{b}}\right) .{ }^{13} \mathrm{C}-\mathrm{NMR}\left(150 \mathrm{MHz}\right.$, acetone- $\left.d_{6}\right) \delta(\mathrm{ppm}): 163.47$, $160.65,159.44,158.46,154.85(\mathrm{C}=\mathrm{NOH}), 131.72,128.80,116.12,99.24,96.11,94.64,77.39,55.67,30.28$. HR ESI-MS $m / z$ calculated for $\mathrm{C}_{16} \mathrm{H}_{15} \mathrm{NO}_{5}[\mathrm{M}+\mathrm{H}]^{+}$302.1023, found $[\mathrm{M}+\mathrm{H}]^{+} 302.1031$.

7,4'-Di-O-methylnaringenin oxime (2b), Yield 80.8\% (0.424 g), white powder, m.p. $155-157{ }^{\circ} \mathrm{C} .{ }^{1} \mathrm{H}-\mathrm{NMR}$ $\left(600 \mathrm{MHz}\right.$, acetone- $\left.d_{6}\right) \delta(\mathrm{ppm}): 11.03(\mathrm{~s}, 1 \mathrm{H}, \mathrm{NOH}), 10.41(\mathrm{~s}, 1 \mathrm{H}, \mathrm{OH}-5), 7.51-7.43\left(\mathrm{~m}, 2 \mathrm{H}, \mathrm{AA}^{\prime} \mathrm{BB}^{\prime}\right.$, H-2' ${ }^{\prime}$ H-6 $\left.{ }^{\prime}\right), 7.03-6.96\left(\mathrm{~m}, 2 \mathrm{H}, \mathrm{AA}^{\prime} \mathrm{BB}^{\prime}, \mathrm{H}^{\prime} 3^{\prime}, \mathrm{H}-5^{\prime}\right), 6.06$ (d, $\left.J=2.5 \mathrm{~Hz}, 1 \mathrm{H}, \mathrm{H}-6\right), 6.05(\mathrm{~d}, J=2.5 \mathrm{~Hz}, 1 \mathrm{H}$, $\mathrm{H}-8), 5.12(\mathrm{dd}, J=11.9,3.3 \mathrm{~Hz}, 1 \mathrm{H}, \mathrm{H}-2), 3.82\left(\mathrm{~s}, 3 \mathrm{H},-\mathrm{CH}_{3}\right), 3.76\left(\mathrm{~s}, 3 \mathrm{H},-\mathrm{CH}_{3}\right), 3.48(\mathrm{dd}, J=17.0,3.3$ $\left.\mathrm{Hz}, 1 \mathrm{H}, \mathrm{H}-3_{\mathrm{a}}\right), 2.81\left(\mathrm{dd}, J=17.0,11.9 \mathrm{~Hz}, 1 \mathrm{H}, \mathrm{H}-3_{\mathrm{b}}\right) .{ }^{13} \mathrm{C}-\mathrm{NMR}\left(150 \mathrm{MHz}\right.$, acetone- $\left.d_{6}\right) \delta(\mathrm{ppm}): 163.49$, $160.74,160.65,159.34,154.73(\mathrm{C}=\mathrm{NOH}), 132.85,128.68,114.72,99.25,96.14,94.67,77.22,55.69,55.59$, 30.26. HR ESI-MS $m / z$ calculated for $\mathrm{C}_{17} \mathrm{H}_{17} \mathrm{NO}_{5}[\mathrm{M}+\mathrm{H}]^{+} 316.1179$, found $[\mathrm{M}+\mathrm{H}]^{+} 316.1185$.

5,7,4'-Tri-O-methylnaringenin oxime (3b), Yield 96.0\% (0.302 g), white powder, m.p. $211-214{ }^{\circ} \mathrm{C}$, lit. 200-202 ${ }^{\circ} \mathrm{C}$ [22]. ${ }^{1} \mathrm{H}-\mathrm{NMR}\left(600 \mathrm{MHz}, \mathrm{DMSO}-d_{6}\right) \delta(\mathrm{ppm}): 11.05$ (s, $\left.1 \mathrm{H}, \mathrm{NOH}\right), 7.42-7.37$ (m, 2H, $\mathrm{AA}^{\prime} \mathrm{BB}^{\prime}$,

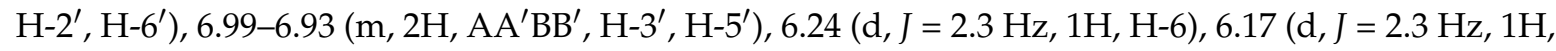
$\mathrm{H}-8), 5.03(\mathrm{dd}, J=11.7,3.3 \mathrm{~Hz}, 1 \mathrm{H}, \mathrm{H}-2), 3.76\left(\mathrm{~s}, 3 \mathrm{H},-\mathrm{CH}_{3}\right), 3.75\left(\mathrm{~s}, 3 \mathrm{H},-\mathrm{CH}_{3}\right), 3.74\left(\mathrm{~s}, 3 \mathrm{H},-\mathrm{CH}_{3}\right), 3.33$ $\left(\mathrm{dd}, J=16.9,3.3 \mathrm{~Hz}, 1 \mathrm{H}, \mathrm{H}-3_{\mathrm{a}}\right), 2.69$ (dd, $\left.J=16.9,11.7 \mathrm{~Hz}, 1 \mathrm{H}, \mathrm{H}-3_{\mathrm{b}}\right) .{ }^{13} \mathrm{C}-\mathrm{NMR}\left(150 \mathrm{MHz}, \mathrm{DMSO}-d_{6}\right)$ $\delta$ (ppm): 160.85, 159.21, 159.1, 158.6, $147.66(\mathrm{C}=\mathrm{NOH}), 131.97,127.82,113.77,101.88,94.28,93.24$, $75.95,55.66,55.29,55.12,30.14$. HR ESI-MS $m / z$ calculated for $\mathrm{C}_{18} \mathrm{H}_{19} \mathrm{NO}_{5}[\mathrm{M}+\mathrm{H}]^{+} 330.1336$, found $[\mathrm{M}+\mathrm{H}]^{+} 330.1338$, lit. HR ESI-MS $m / z$ calculated for $\mathrm{C}_{18} \mathrm{H}_{19} \mathrm{NO}_{5}[\mathrm{M}+\mathrm{H}]^{+} 330.1341$, found $[\mathrm{M}+\mathrm{H}]^{+}$ 330.1335 [22].

7-O-Ethylnaringenin oxime $(4 \mathbf{b})$, Yield $96.9 \%(0.509 \mathrm{~g})$, white powder, m.p. $203-205{ }^{\circ} \mathrm{C} .{ }^{1} \mathrm{H}-\mathrm{NMR}$ $\left(600 \mathrm{MHz}\right.$, acetone- $\left.d_{6}\right) \delta(\mathrm{ppm}): 11.01(\mathrm{~s}, 1 \mathrm{H}, \mathrm{NOH}), 10.38(\mathrm{~s}, 1 \mathrm{H}, \mathrm{OH}-5), 8.47\left(\mathrm{~s}, 1 \mathrm{H}, \mathrm{OH}-4^{\prime}\right), 7.41-7.34$ $\left(\mathrm{m}, 2 \mathrm{H}, \mathrm{AA}^{\prime} \mathrm{BB}^{\prime}, \mathrm{H}-2^{\prime}, \mathrm{H}^{\prime} 6^{\prime}\right), 6.92-6.85\left(\mathrm{~m}, 2 \mathrm{H}, \mathrm{AA}^{\prime} \mathrm{BB}^{\prime}, \mathrm{H}^{\prime} 3^{\prime}, \mathrm{H}-5^{\prime}\right), 6.04(\mathrm{~d}, J=2.4 \mathrm{~Hz}, 1 \mathrm{H}, \mathrm{H}-6), 6.02$ $(\mathrm{d}, J=2.4 \mathrm{~Hz}, 1 \mathrm{H}, \mathrm{H}-8), 5.07(\mathrm{dd}, J=12.0,3.2 \mathrm{~Hz}, 1 \mathrm{H}, \mathrm{H}-2), 4.02\left(\mathrm{q}, J=7.0 \mathrm{~Hz}, 2 \mathrm{H},-\mathrm{CH}_{2}-\right), 3.46(\mathrm{dd}$, $\left.J=17.1,3.2 \mathrm{~Hz}, 1 \mathrm{H}, \mathrm{H}-3_{\mathrm{a}}\right), 2.79\left(\mathrm{dd}, J=17.1,12.0 \mathrm{~Hz}, 1 \mathrm{H}, \mathrm{H}-3_{\mathrm{b}}\right), 1.33\left(\mathrm{t}, J=7.0 \mathrm{~Hz}, 3 \mathrm{H},-\mathrm{CH}_{3}\right) .{ }^{13} \mathrm{C}-\mathrm{NMR}$ $\left(150 \mathrm{MHz}\right.$, acetone- $\left.d_{6}\right) \delta(\mathrm{ppm}): 162.78,160.63,159.42,158.46,154.87(\mathrm{C}=\mathrm{NOH}), 131.75,128.80,116.12$, 99.15, 96.54, 95.07, 77.38, 64.16, 30.30, 14.97. HR ESI-MS $m / z$ calculated for $\mathrm{C}_{17} \mathrm{H}_{17} \mathrm{NO}_{5}[\mathrm{M}+\mathrm{H}]^{+}$ 316.1179, found $[\mathrm{M}+\mathrm{H}]^{+}$316.1191.

7,4'-Di-O-ethylnaringenin oxime (5b), Yield 83.8\% (0.307 g), white powder, m.p. $160-162{ }^{\circ} \mathrm{C} .{ }^{1} \mathrm{H}-\mathrm{NMR}$ $\left(600 \mathrm{MHz}\right.$, acetone- $\left.d_{6}\right) \delta(\mathrm{ppm}): 11.01(\mathrm{~s}, 1 \mathrm{H}, \mathrm{NOH}), 10.38(\mathrm{~s}, 1 \mathrm{H}, \mathrm{OH}-5), 7.48-7.41\left(\mathrm{~m}, 2 \mathrm{H}, \mathrm{AA}^{\prime} \mathrm{BB}^{\prime}\right.$, H-2' $\left.{ }^{\prime} \mathrm{H}-6^{\prime}\right), 7.00-6.91\left(\mathrm{~m}, 2 \mathrm{H}, \mathrm{AA}^{\prime} \mathrm{BB}^{\prime}, \mathrm{H}^{\prime} 3^{\prime}, \mathrm{H}-5^{\prime}\right), 6.04(\mathrm{~d}, J=2.3 \mathrm{~Hz}, 1 \mathrm{H}, \mathrm{H}-6), 6.03(\mathrm{~d}, J=2.3 \mathrm{~Hz}, 1 \mathrm{H}$, $\mathrm{H}-8), 5.11(\mathrm{dd}, J=11.9,3.2 \mathrm{~Hz}, 1 \mathrm{H}, \mathrm{H}-2), 4.07\left(\mathrm{q}, J=7.0 \mathrm{~Hz}, 2 \mathrm{H},-\mathrm{CH}_{2}-\right), 4.02\left(\mathrm{q}, J=7.0 \mathrm{~Hz}, 2 \mathrm{H},-\mathrm{CH}_{2}-\right)$, $3.47\left(\mathrm{dd}, J=17.1,3.2 \mathrm{~Hz}, 1 \mathrm{H}, \mathrm{H}-3_{\mathrm{a}}\right), 2.81\left(\mathrm{dd}, J=17.1,11.9 \mathrm{~Hz}, 1 \mathrm{H}, \mathrm{H}-3_{\mathrm{b}}\right), 1.37\left(\mathrm{t}, J=7.0 \mathrm{~Hz}, 3 \mathrm{H},-\mathrm{CH}_{3}\right)$, $1.34\left(\mathrm{t}, J=7.0 \mathrm{~Hz}, 3 \mathrm{H},-\mathrm{CH}_{3}\right) .{ }^{13} \mathrm{C}-\mathrm{NMR}\left(150 \mathrm{MHz}\right.$, acetone- $\left.d_{6}\right) \delta(\mathrm{ppm}): 162.80,160.63,160.06,159.33$, $154.76(\mathrm{C}=\mathrm{NOH}), 132.73,128.67,115.23,99.15,96.57,95.10,77.22,64.17,64.05,30.27,15.10,14.97$. HR ESI-MS $m / z$ calculated for $\mathrm{C}_{19} \mathrm{H}_{21} \mathrm{NO}_{5}[\mathrm{M}+\mathrm{H}]^{+} 344.1492$, found $[\mathrm{M}+\mathrm{H}]^{+} 344.1502$.

5,7,4'-Tri-O-ethylnaringenin oxime (6b), Yield 95.5\% (0.149 g), white powder, m.p. $169-171^{\circ} \mathrm{C} .{ }^{1} \mathrm{H}-\mathrm{NMR}$ $\left(600 \mathrm{MHz}, \mathrm{DMSO}-d_{6}\right) \delta(\mathrm{ppm}): 10.89(\mathrm{~s}, 1 \mathrm{H}, \mathrm{NOH}), 7.38\left(\mathrm{~d}, J=8.2 \mathrm{~Hz}, 2 \mathrm{H}, \mathrm{H}-2^{\prime}, \mathrm{H}-6^{\prime}\right), 6.93(\mathrm{~d}, J=8.2 \mathrm{~Hz}$, 2H, H-3', H-5'), 6.19 (s, 1H, H-6), 6.13 (s, 1H, H-8), 5.00 (d, J = 11.7 Hz, 1H, H-2), 4.11-3.99 (m, 6H, 3x- $\mathrm{CH}_{2}-$ ), $3.31(\mathrm{~d}, J=16.8 \mathrm{~Hz}, 1 \mathrm{H}, \mathrm{H}-3 \mathrm{a}), 2.68(\mathrm{dd}, J=16.8,11.7 \mathrm{~Hz}, 1 \mathrm{H}, \mathrm{H}-3 \mathrm{~b}), 1.35-1.27\left(\mathrm{~m}, 9 \mathrm{H}, 3 \mathrm{x}-\mathrm{CH}_{3}\right) .{ }^{13} \mathrm{C}-\mathrm{NMR}$ 
(150 MHz, DMSO- $\left.d_{6}\right) \delta$ (ppm): 159.99, 158.70, 158.36, 158.30, 147.78 (C=NOH), 131.88, 127.81, 114.20, 102.26, $94.68,94.64,76.01,63.87,63.16,63.02,30.33,14.63,14.60,14.53$. HR ESI-MS $m / z$ calculated for $\mathrm{C}_{21} \mathrm{H}_{25} \mathrm{NO}_{5}$ $[\mathrm{M}+\mathrm{H}]^{+} 372.1805$, found $[\mathrm{M}+\mathrm{H}]^{+} 372.1812$.

7-O-Pentylnaringenin oxime (7b), Yield 86.2\% (0.450 g), white powder, m.p. $189-191{ }^{\circ} \mathrm{C} .{ }^{1} \mathrm{H}-\mathrm{NMR}$ $\left(600 \mathrm{MHz}\right.$, acetone- $\left.d_{6}\right) \delta(\mathrm{ppm}): 11.01(\mathrm{~s}, 1 \mathrm{H}, \mathrm{NOH}), 10.37(\mathrm{~s}, 1 \mathrm{H}, \mathrm{OH}-5), 8.46\left(\mathrm{~s}, 1 \mathrm{H}, \mathrm{OH}-4^{\prime}\right), 7.42-7.34$ $\left(\mathrm{m}, 2 \mathrm{H}, \mathrm{AA}^{\prime} \mathrm{BB}^{\prime}, \mathrm{H}-2^{\prime}, \mathrm{H}-6^{\prime}\right), 6.93-6.86\left(\mathrm{~m}, 2 \mathrm{H}, \mathrm{AA}^{\prime} \mathrm{BB}^{\prime}, \mathrm{H}-3^{\prime}, \mathrm{H}-5^{\prime}\right), 6.07(\mathrm{~d}, J=2.5 \mathrm{~Hz}, 1 \mathrm{H}, \mathrm{H}-6), 6.06(\mathrm{~d}$, $J=2.5 \mathrm{~Hz}, 1 \mathrm{H}, \mathrm{H}-8), 5.07(\mathrm{dd}, J=12.0,3.2 \mathrm{~Hz}, 1 \mathrm{H}, \mathrm{H}-2), 3.96\left(\mathrm{t}, J=6.6 \mathrm{~Hz}, 2 \mathrm{H},-\mathrm{CH}_{2}-\right), 3.46(\mathrm{dd}, J=17.1$, $3.2 \mathrm{~Hz}, 1 \mathrm{H}, \mathrm{H}-3_{\mathrm{a}}$ ), 2.79 (dd, J = 17.1, $\left.12.0 \mathrm{~Hz}, 1 \mathrm{H}, \mathrm{H}-3_{\mathrm{b}}\right), 1.74\left(\mathrm{p}, J=6.6 \mathrm{~Hz}, 2 \mathrm{H},-\mathrm{CH}_{2}-\right), 1.47-1.33$ (m, $\left.4 \mathrm{H}, 2 \mathrm{x}-\mathrm{CH}_{2}-\right), 0.92\left(\mathrm{t}, J=7.2 \mathrm{~Hz}, 3 \mathrm{H},-\mathrm{CH}_{3}\right) .{ }^{13} \mathrm{C}-\mathrm{NMR}\left(150 \mathrm{MHz}\right.$, acetone- $\left.d_{6}\right) \delta(\mathrm{ppm}): 162.95,160.63$, $159.42,158.45,154.87$ (C=NOH), 131.76, 128.79, 116.12, 99.13, 96.58, 95.11, 77.37, 68.64, 30.30, 30.06, $28.89,23.07,14.29$. HR ESI-MS $m / z$ calculated for $\mathrm{C}_{20} \mathrm{H}_{23} \mathrm{NO}_{5}[\mathrm{M}+\mathrm{H}]^{+} 358.1649$, found $[\mathrm{M}+\mathrm{H}]^{+}$ 358.1651 .

7,4'-Di-O-pentylnaringenin oxime (8b), Yield 99.5\% (0.474 g), white powder, m.p. $71-74{ }^{\circ} \mathrm{C} .{ }^{1} \mathrm{H}-\mathrm{NMR}$ $\left(600 \mathrm{MHz}\right.$, acetone- $\left.d_{6}\right) \delta(\mathrm{ppm}): 11.00(\mathrm{~s}, 1 \mathrm{H}, \mathrm{NOH}), 10.38(\mathrm{~s}, 1 \mathrm{H}, \mathrm{OH}-5), 7.49-7.42\left(\mathrm{~m}, 2 \mathrm{H}, \mathrm{AA}^{\prime} \mathrm{BB}^{\prime}\right.$, H-2' ${ }^{\prime}$ H-6 $\left.{ }^{\prime}\right), 7.01-6.95\left(\mathrm{~m}, 2 \mathrm{H}, \mathrm{AA}^{\prime} \mathrm{BB}^{\prime}, \mathrm{H}-3^{\prime}, \mathrm{H}^{\prime} 5^{\prime}\right), 6.08$ (d, J = 2.4 Hz, 1H, H-6), 6.06 (d, J = 2.4 Hz, 1H, $\mathrm{H}-8), 5.11(\mathrm{dd}, J=11.9,3.2 \mathrm{~Hz}, 1 \mathrm{H}, \mathrm{H}-2), 4.02\left(\mathrm{t}, J=6.5 \mathrm{~Hz}, 2 \mathrm{H},-\mathrm{CH}_{2}-\right), 3.96\left(\mathrm{t}, J=6.5 \mathrm{~Hz}, 2 \mathrm{H},-\mathrm{CH}_{2}-\right)$, $3.47\left(\mathrm{dd}, J=17.1,3.2 \mathrm{~Hz}, 1 \mathrm{H}, \mathrm{H}-3_{\mathrm{a}}\right), 2.81\left(\mathrm{dd}, J=17.1,11.9 \mathrm{~Hz}, 1 \mathrm{H}, \mathrm{H}-3_{\mathrm{b}}\right), 1.82-1.70\left(\mathrm{~m}, 4 \mathrm{H}, 2 \mathrm{x}-\mathrm{CH}_{2}-\right)^{2}$, 1.48-1.36 (m, 8H, 4x- $\left.\mathrm{CH}_{2}-\right), 0.93\left(\mathrm{t}, J=7.4 \mathrm{~Hz}, 3 \mathrm{H},-\mathrm{CH}_{3}\right), 0.92\left(\mathrm{t}, J=7.4 \mathrm{~Hz}, 3 \mathrm{H},-\mathrm{CH}_{3}\right) .{ }^{13} \mathrm{C}-\mathrm{NMR}(150$ $\mathrm{MHz}$, acetone- $\left.d_{6}\right) \delta(\mathrm{ppm}): 162.08,159.75,159.35,158.44,153.88(\mathrm{C}=\mathrm{NOH}), 131.84,127.78,114.38,98.26$, $95.72,94.25,76.34,67.77,67.70,29.39,29.05,28.93,28.08,28.01,22.24,22.19,13.43,13.41$. HR ESI-MS $m / z$ calculated for $\mathrm{C}_{25} \mathrm{H}_{33} \mathrm{NO}_{5}[\mathrm{M}+\mathrm{H}]^{+} 428.2432$, found $[\mathrm{M}+\mathrm{H}]^{+} 428.2436$.

7-O-Dodecylnaringenin oxime (9b), Yield 95.2\% (0.197 g), white powder, m.p. $156-159{ }^{\circ} \mathrm{C} .{ }^{1} \mathrm{H}-\mathrm{NMR}$ $\left(600 \mathrm{MHz}\right.$, acetone- $\left.d_{6}\right) \delta(\mathrm{ppm}): 11.01(\mathrm{~s}, 1 \mathrm{H}, \mathrm{NOH}), 10.37(\mathrm{~s}, 1 \mathrm{H}, \mathrm{OH}-5), 8.47\left(\mathrm{~s}, 1 \mathrm{H}, \mathrm{OH}-4^{\prime}\right), 7.41-7.34$ $\left(\mathrm{m}, 2 \mathrm{H}, \mathrm{AA}^{\prime} \mathrm{BB}^{\prime}, \mathrm{H}-2^{\prime}, \mathrm{H}-6^{\prime}\right), 6.92-6.86\left(\mathrm{~m}, 2 \mathrm{H}, \mathrm{AA}^{\prime} \mathrm{BB}^{\prime}, \mathrm{H}-3^{\prime}, \mathrm{H}-5^{\prime}\right), 6.05(\mathrm{~d}, J=2.5 \mathrm{~Hz}, 1 \mathrm{H}, \mathrm{H}-6), 6.03(\mathrm{~d}$, $J=2.5 \mathrm{~Hz}, 1 \mathrm{H}, \mathrm{H}-8), 5.07(\mathrm{dd}, J=12.0,3.1 \mathrm{~Hz}, 1 \mathrm{H}, \mathrm{H}-2), 3.97\left(\mathrm{t}, J=6.6 \mathrm{~Hz}, 2 \mathrm{H},-\mathrm{CH}_{2}-\right), 3.46(\mathrm{dd}, J=17.1$, $\left.3.1 \mathrm{~Hz}, 1 \mathrm{H}, \mathrm{H}-3_{\mathrm{a}}\right), 2.79\left(\mathrm{dd}, J=17.1,12.0 \mathrm{~Hz}, 1 \mathrm{H}, \mathrm{H}-3_{\mathrm{b}}\right), 1.74\left(\mathrm{p}, J=6.7 \mathrm{~Hz}, 2 \mathrm{H},-\mathrm{CH}_{2}-\right), 1.49-1.41(\mathrm{~m}, 2 \mathrm{H}$, $\left.-\mathrm{CH}_{2}-\right), 1.38-1.22\left(\mathrm{~m}, 16 \mathrm{H}, 8 \mathrm{x}-\mathrm{CH}_{2}-\right), 0.87\left(\mathrm{t}, J=7.0 \mathrm{~Hz}, 3 \mathrm{H},-\mathrm{CH}_{3}\right) .{ }^{13} \mathrm{C}-\mathrm{NMR}\left(150 \mathrm{MHz}\right.$, acetone- $\left.d_{6}\right)$ $\delta$ (ppm): 162.95, 160.62, 159.41, 158.45, 154.87 (C=NOH), 131.76, 128.79, 116.11, 99.12, 96.58, 95.11, 77.37, 68.65, 32.63, 30.38, 30.36, 30.31, 30.07, 30.05, 26.70, 23.33, 14.36. HR ESI-MS $m / z$ calculated for $\mathrm{C}_{27} \mathrm{H}_{37} \mathrm{NO}_{5}[\mathrm{M}+\mathrm{H}]^{+} 456.2745$, found $[\mathrm{M}+\mathrm{H}]^{+} 456.2767$.

7,4'-Di-O-dodecylnaringenin oxime (10b), Yield $86.7 \%$ ( $0.107 \mathrm{~g})$, white powder, m.p. $82-85{ }^{\circ} \mathrm{C} .{ }^{1} \mathrm{H}-\mathrm{NMR}$ $\left(600 \mathrm{MHz}\right.$, acetone- $\left.d_{6}\right) \delta(\mathrm{ppm}): 11.01(\mathrm{~s}, 1 \mathrm{H}, \mathrm{NOH}), 10.39(\mathrm{~s}, 1 \mathrm{H}, \mathrm{OH}-5), 7.48-7.43\left(\mathrm{~m}, 2 \mathrm{H}, \mathrm{AA}^{\prime} \mathrm{BB}^{\prime}\right.$ H-2' $\left.{ }^{\prime} \mathrm{H}-6^{\prime}\right), 7.01-6.95\left(\mathrm{~m}, 2 \mathrm{H}, \mathrm{AA}^{\prime} \mathrm{BB}^{\prime}, \mathrm{H}^{\prime} 3^{\prime}, \mathrm{H}-5^{\prime}\right), 6.05(\mathrm{~d}, J=2.4 \mathrm{~Hz}, 1 \mathrm{H}, \mathrm{H}-6), 6.04(\mathrm{~d}, J=2.4 \mathrm{~Hz}$, $1 \mathrm{H}, \mathrm{H}-8), 5.11(\mathrm{dd}, J=11.8,3.2 \mathrm{~Hz}, 1 \mathrm{H}, \mathrm{H}-2), 4.03\left(\mathrm{t}, J=6.6 \mathrm{~Hz}, 2 \mathrm{H},-\mathrm{CH}_{2}-\right), 3.97(\mathrm{t}, J=6.6 \mathrm{~Hz}, 2 \mathrm{H}$, $\left.-\mathrm{CH}_{2}-\right), 3.47\left(\mathrm{dd}, J=17.0,3.2 \mathrm{~Hz}, 1 \mathrm{H}, \mathrm{H}-3_{\mathrm{a}}\right), 2.79\left(\mathrm{dd}, J=17.0,11.8 \mathrm{~Hz}, 1 \mathrm{H}, \mathrm{H}-3_{\mathrm{b}}\right), 1.82-1.71(\mathrm{~m}, 4 \mathrm{H}$, 2x- $\left.\mathrm{CH}_{2}-\right), 1.53-1.42\left(\mathrm{~m}, 4 \mathrm{H}, 2 \mathrm{x}-\mathrm{CH}_{2}-\right), 1.40-1.26\left(\mathrm{~m}, 32 \mathrm{H}, 16 \mathrm{x}-\mathrm{CH}_{2}-\right), 0.88\left(\mathrm{t}, J=7.0 \mathrm{~Hz}, 3 \mathrm{H},-\mathrm{CH}_{3}\right)$, $0.88\left(\mathrm{t}, J=7.0 \mathrm{~Hz}, 3 \mathrm{H},-\mathrm{CH}_{3}\right) .{ }^{13} \mathrm{C}-\mathrm{NMR}\left(150 \mathrm{MHz}\right.$, acetone- $\left.d_{6}\right) \delta(\mathrm{ppm}): 162.96,160.63,160.23,159.32$, 154.75 (C=NOH), 132.71, 128.65, 115.26, 99.13, 96.61, 95.13, 77.22, 68.66, 68.58, 32.64, 30.38, 30.37, 30.35, $30.33,30.32,30.28,30.06,26.78,26.70,23.34,14.37$. HR ESI-MS $m / z$ calculated for $\mathrm{C}_{39} \mathrm{H}_{61} \mathrm{NO}_{5}[\mathrm{M}+\mathrm{H}]^{+}$ 624.4623, found $[\mathrm{M}+\mathrm{H}]^{+}$624.4613.

\subsection{Biological Activity}

Antimicrobial activity was performed on two strains of bacteria: E. coli ATCC10536 and S. aureus DSM799 and four strains of fungi: C. albicans DSM1386, F. linii KB-F1, A. alternata CBS1526 and A. niger DSM1957. All the microorganisms were from the collection of the Faculty of Biotechnology and Food Microbiology, Wroclaw University of Environmental and Life Sciences. The culture medium for bacteria was nutrient broth (Biocorp, Warsaw, Poland), and that for fungi was YM medium, which 
consisted of $3 \mathrm{~g}$ yeast extract, $3 \mathrm{~g}$ malt extract, $5 \mathrm{~g}$ bacteriological peptone and $10 \mathrm{~g}$ of glucose dissolved in $1 \mathrm{~L}$ of distilled water. Tests were prepared on 100-well microtiter plates, with the working volume in each well being $300 \mu \mathrm{L}: 280 \mu \mathrm{L}$ of culture medium, $10 \mu \mathrm{L}$ of microorganism suspension and $10 \mu \mathrm{L}$ of naringenin derivative dissolved in dimethyl sulfoxide $(0.3 \%(w / v))$. The final concentration of the derivative was $0.1 \%(w / v)$. Each culture was carried out in 3 replications. The optical density of the cell suspension was measured on Bioscreen C (Automated Growth Curve Analysis System Lab System, Helsinki, Finland) at $560 \mathrm{~nm}$ automatically, at regular intervals of $30 \mathrm{~min}$ for 2-3 days. Cell cultures were maintained at $28^{\circ} \mathrm{C}$ on a continuous shaker. To prepare the growth curves for each strain, the mean values of the absorbance of the medium as a function of time were used. The resulting antimicrobial activity was expressed as the increase in optical density $(\Delta \mathrm{OD})$ and was compared to that of the control cultures in the medium supplemented with dimethyl sulfoxide.

\section{Conclusions}

In this paper, we report the synthesis and evaluation of the antimicrobial activity of the $O$-alkyl derivatives of naringenin and their oximes including novel compounds $\mathbf{7 a - 1 0 a}, \mathbf{2} \mathbf{b}$, and $\mathbf{4 b} \mathbf{b} \mathbf{- 1 0 b}$.

The highest inhibitory effect against E. coli ATCC10536, A. alternata CBS1526, F. linii KB-F1, and $A$. niger DSM1957 was observed for the novel 7,4'-di-O-pentylnaringenin (8a). Moreover, 7-O-dodecylnaringenin (9a) prevented the growth of E. coli ATCC10536. Furthermore, compound 10a, which has one more dodecyl group attached at position $C-4^{\prime}$, presented the same activity against $F$. linii KB-F1. Introduction of the oxime group afforded 8 new derivatives, which were never described in the literature. The best inhibitory effect was observed for the novel 7-O-dodecylnaringenin oxime (9b). Our results showed that elongation of the O-alkyl groups attached to positions C-7 and C- $4^{\prime}$ in naringenin increases the antimicrobial activity. Moreover, replacement of carbonyl with the oxime group enhanced the inhibitory effect, especially the antifungal activity.

Supplementary Materials: The following are available online.

Acknowledgments: Publication supported by the National Science Centre, Grant No. 2016/21/B/NZ9/01904 and the Wroclaw Centre of Biotechnology under the Leading National Research Centre (KNOW) programme for years 2014-2018.

Author Contributions: Joanna Kozłowska and Barłłomiej Potaniec conceived and designed the experiments; Joanna Kozłowska performed the experiments and prepared the supplementary materials; Joanna Kozłowska and Bartłomiej Potaniec analyzed the data and interpreted the NMR spectra; Barbara Żarowska measured the antimicrobial activity; Joanna Kozłowska and Mirosław Anioł wrote the paper.

Conflicts of Interest: The authors declare no conflict of interest.

\section{References}

1. Tripoli, E.; Guardia, M.; Giammanco, S.; Majo, D.; Giammanco, M. Citrus flavonoids: Molecular structure, biological activity and nutritional properties: A review. Food Chem. 2007, 104, 466-479. [CrossRef]

2. Walle, T. Absorption and metabolism of flavonoids. Free Radic. Biol. Med. 2004, 36, 829-837. [CrossRef] [PubMed]

3. Erlund, I. Review of the flavonoids quercetin, hesperetin, and naringenin. Dietary sources, bioactivities, bioavailability, and epidemiology. Nutr. Res. 2004, 24, 851-874. [CrossRef]

4. Fuhr, U.; Klittich, K.; Stain, A.H. Inhibitory effect of grapefruit juice and its bitter principal, naringenin, on CYP1A2 dependent metabolism of caffeine in man. Br. J. Clin. Pharma. 1993, 35, 431-436. [CrossRef]

5. Woo, Y.; Shin, S.Y.; Hyun, J.; Lee, S.D.; Lee, Y.H.; Lim, Y. Flavanones inhibit the clonogenicity of HCT116 cololectal cancer cells. Int. J. Mol. Med. 2012, 29, 403-408. [CrossRef] [PubMed]

6. Hoang, T.K.D.; Huynh, T.K.C.; Nguyen, R.D. Synthesis, characterization, anti-inflammatory and anti-proliferative activity against MCF-7 cells of $O$-alkyl and $O$-acyl flavonoid derivatives. Bioorg. Chem. 2015, 63, 45-52. [CrossRef] [PubMed] 
7. Silva, S.A.S.; Fatima Agra, M.; Tavares, J.F.; Cunha, E.V.L.; Barbosa-Filho, J.M.; Silva, M.S. Flavanones from aerial parts of Cordia globosa (Jacq.) Kunth, Boraginaceae. Rev. Bras. Farmacogn. Braz. J. Pharmacogn. 2010, 20, 682-685. [CrossRef]

8. Nobakht, M.; Grkovic, T.; Trueman, S.J.; Wallace, H.M.; Katouli, M.; Quinn, R.J.; Brooks, P.R. Chemical Constituents of Kino Extract from Corymbia torelliana. Molecules 2014, 19, 17862-17871. [CrossRef] [PubMed]

9. Drira, R.; Sakamoto, K. Sakuranetin Induces Melanogenesis in B16BL6 Melanoma Cells through Inhibition of ERK and PI3K/AKT Signaling Pathways. Phytother. Res. 2016, 30, 997-1002. [CrossRef] [PubMed]

10. Sakoda, C.P.P.; Toledo, C.A.; Perini, A.; Pinheiro, N.M.; Hiyane, M.I.; Santos Grecco, S.; Fátima Lopes Calvo Tibério, I.; Câmara, N.O.S.; Arruda Martins, M.; Lago, J.H.G.; et al. Sakuranetin reverses vascular peribronchial and lung parenchyma remodeling in a murine model of chronic allergic pulmonary inflammation. Acta Histochem. 2016, 118, 615-624. [CrossRef] [PubMed]

11. Lee, E.R.; Kang, Y.J.; Kim, H.J.; Choi, H.Y.; Kang, G.H.; Kim, J.H.; Kim, B.W.; Jeong, H.S.; Park, Y.S.; Cho, S.G. Regulation of Apoptosis by Modified Naringenin Derivatives in Human Colorectal Carcinoma RKO Cells. J. Cell. Biochem. 2008, 104, 259-273. [CrossRef] [PubMed]

12. Lee, K.A.; Moon, S.H.; Lee, J.Y.; Kim, K.T.; Park, Y.S.; Paik, H.D. Antibacterial Activity of a Novel Flavonoid, 7-O-Butyl Naringenin, against Methicillin-Resistant Staphylococcus aureus (MRSA). Food Sci. Biotechnol. 2013, 22, 1725-1728. [CrossRef]

13. Emami, S.; Falahati, M.; Banifatemi, A.; Amanlou, M.; Shafiee, A. (E)- and (Z)-1,2,4-Triazolylchromanone oxime ethers as conformationally constrained antifungals. Bioorg. Med. Chem. 2004, 12, 3971-3976. [CrossRef] [PubMed]

14. Emami, S.; Falahati, M.; Banifatemi, A.; Moshiri, K.; Shafiee, A. Stereoselective Synthesis and in Vitro Antifungal Evaluation of (E)- and (Z)-Imidazolylchromanone Oxime Ethers. Arch. Pharm. Pharm. Med. Chem. 2002, 7, 318-324. [CrossRef]

15. Türkkan, B.; Özyürek, M.; Bener, M.; Güclü, K.; Apak, R. Synthesis, characterization and antioxidant capacity of naringenin-oxime. Spectrochim. Acta A 2012, 85, 235-240. [CrossRef] [PubMed]

16. Özyurek, M.; Akpınar, D.; Bener, M.; Türkkan, B.; Güclü, K.; Apak, R. Novel oxime based flavanone, naringin-oxime: Synthesis, characterization and screening for antioxidant activity. Chem.-Biol. Interact. 2014, 212, 40-46. [CrossRef] [PubMed]

17. Potaniec, B.; Grabarczyk, M.; Stompor, M.; Szumny, A.; Zieliński, P.; Żołnierczyk, A.K.; Anioł, M. Antioxidant activity and spectroscopic data of isoxanthohumol oxime and related compounds. Spectrochim. Acta A 2014, 118, 716-723. [CrossRef] [PubMed]

18. Kocyigit, A.; Koyuncu, I.; Dikilitas, M.; Bahadori, F.; Turkkan, B. Cytotoxic, genotoxic and apoptotic effects of naringenin-oxime relative to naringenin on normal and cancer cell lines. Asian Pac. J. Trop. Biomed. 2016, 6, 872-880. [CrossRef]

19. Yoon, H.; Kim, T.W.; Shin, S.Y.; Park, M.J.; Yong, Y.; Kim, D.W.; Islam, T.; Lee, Y.H.; Jung, K.Y.; Lim, Y. Design, synthesis and inhibitory activities of naringenin derivatives on human colon cancer cells. Bioorg. Med. Chem. Lett. 2013, 23, 232-238. [CrossRef] [PubMed]

20. Chiang, C.Y.; Wang, T.C.; Lee, C.H.; Chen, C.S.; Wang, S.H.; Lin, Y.C.; Juang, S.H. WTC-01, a novel synthetic oxime-flavone compound, destabilizes microtubules in human nasopharyngeal carcinoma cells in vitro and in vivo. Br. J. Pharmacol. 2015, 172, 4671-4683. [CrossRef] [PubMed]

21. Wang, T.C.; Chen, I.L.; Lu, C.M.; Kuo, D.H.; Liao, C.H. Synthesis, and Cytotoxic and Antiplatelet of Oximeand Methyloxime-Containing Flavone, Isoflavone, and Xanthone Derivatives. Chem. Biodivers. 2005, 2, 253-263. [CrossRef] [PubMed]

22. Yenjai, C.; Wanich, S. Cytotoxicity against KB and NCI-H187 cell lines of modified flavonoids from Kaempferia parviflora. Bioorg. Med. Chem. Lett. 2010, 20, 2821-2823. [CrossRef] [PubMed]

23. Cushnie, T.P.T.; Lamb, A.J. Antimicrobial activity of flavonoids. Int. J. Antimicrob. Ag. 2005, 26, $343-356$. [CrossRef]

24. Deka, N.; Mariotte, A.M.; Boumendje, A. Microwave mediated solvent-free acetylation of deactivated and hindered phenols. Green Chem. 2001, 3, 263-264. [CrossRef]

25. Feng, L.; Maddox, M.M.; Alam, M.Z.; Tsutsumi, L.S.; Narula, G.; Bruhn, D.F.; Wu, X.; Sandhaus, S.; Lee, R.B.; Simmons, C.J.; et al. Synthesis, structure-activity relationship studies, and antibacterial evaluation of 4-chromanones and chalcones, as well as olympicin A and derivatives. J. Med. Chem. 2014, 57, 8398-8420. [CrossRef] [PubMed] 
26. Yenjai, C.; Wanich, S.; Pitchuanchom, S.; Sripanidkulchai, B. Structural Modification of 5,7-Dimethoxyflavone from Kaempferia parviflora and biological activities. Arch. Pharm. Res. 2009, 32, 1179-1184. [CrossRef] [PubMed]

27. Suksamrarn, A.; Chotipong, A.; Suavansri, T.; Boongird, S.; Timsuksai, P.; Vimuttipong, S.; Chuaynugul, A. Antimycobacterial activity and cytotoxicity of flavonoids from the flowers of Chromolaena odorata. Arch. Pharm. Res. 2004, 27, 507-511. [CrossRef] [PubMed]

28. Bae, E.A.; Han, M.J.; Kim, D.H. In Vitro Anti-Helicobacter Pylori Activity of Some Flavonoids and Their Metabolites. Planta Med. 1999, 65, 442-443. [CrossRef] [PubMed]

29. Ilboudo, O.; Bonzi, S.; Tapsoba, I.; Somda, I.; Bonzi-Coulibaly, Y.L. In vitro antifungal activity of flavonoid diglycosides of Mentha piperita and their oxime derivatives against two cereals fungi. CR Chim. 2016, 19, 857-862. [CrossRef]

30. Oyama, K.; Kondo, T. Total Synthesis of Flavocommelin, a Component of the Blue Supramolecular Pigment from Commelina communis, on the Basis of Direct 6-C-Gycosylation of Flavan. J. Org. Chem. 2004, 69, 5240-5246. [CrossRef] [PubMed]

31. Righi, G.; Silvestri, I.P.; Barontini, M.; Crisante, F.; Di Manno, A.; Pelagalli, R.; Bovicelli, P. Efficient synthesis of scutellarein. Nat. Prod. Res. 2012, 26, 1278-1284. [CrossRef] [PubMed]

32. Aritomi, M.; Kawasaki, T. Dehydro-para-asebotin, a New Chalcone Glucoside in the Flowers of Gnaphalium affine D. DON. Chem. Pharm. Bull. 1974, 22, 1800-1805. [CrossRef] [PubMed]

Sample Availability: Samples of the compounds naringenin, naringenin oxime, $\mathbf{1 a}-\mathbf{1 0 a}$ and $\mathbf{1 b} \mathbf{- 1 0 b}$ are available from the authors.

(c) 2017 by the authors. Licensee MDPI, Basel, Switzerland. This article is an open access article distributed under the terms and conditions of the Creative Commons Attribution (CC BY) license (http:/ / creativecommons.org/licenses/by/4.0/). 